\title{
N BASICITY OF SUBSTITUTED FULLERO[60]/[70]PYRROLIDINES ACCORDING TO DFT/TD-DFT CALCULATIONS AND CHEMICAL THERMODYNAMICS
}

Ekaterina N. Ovchenkova*1, Arshak A. Tsaturyan*2,3, Nataliya G. Bichan ${ }^{1}$, Tatyana N. Lomova ${ }^{1}$

${ }^{1}$ G.A. Krestov Institute of Solution Chemistry of the Russian Academy of Sciences,

Akademicheskaya Str. 1, Ivanovo, Russia email: enk@isc-ras.ru

${ }^{2}$ Institute of Physical and Organic Chemistry, Southern Federal University, Stachki Av. 194/2

Rostov-on-Don, Russia email: caturyan@sfedu.ru

${ }^{3}$ Department of Chemistry, University of Turin, Via Pietro Giuria 7, 10125, Torino, Italy

Corresponding authors:

Arshak A. Tsaturyan email: caturyan@sfedu.ru Ekaterina N. Ovchenkova enk@isc-ras.ru

Synthesis of 1'-N-methyl-2'-(pyridin-4-yl)pyrrolidino[3',4':1,2][60]fullerene, PyC60 ................ S2 Synthesis of 1'-N-methyl-2'-(1H-imidazol-1-yl)-phenylpyrrolidino[3',4':1,2][60]fullerene, ImC 60

Synthesis of 2'-(Pyridin-4-yl)-5'-(pyridin-2-yl)-1'-(pyridin-2-ylmethyl)-

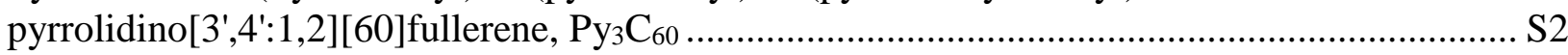

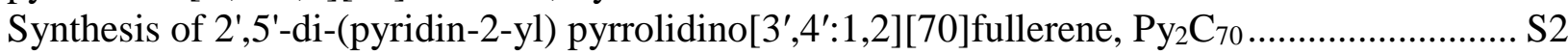

Table S1. The UV-vis spectra of substituted fullero[60]/[70]pyrrolidines in toluene and $\mathrm{CH}_{2} \mathrm{Cl}_{2}$.

Fig. S1. (a) The UV-vis spectra of $\mathrm{ImC}_{60}$ in $\mathrm{CF}_{3} \mathrm{COOH}-\mathrm{CH}_{2} \mathrm{Cl}_{2}$ obtained during titration

$\left(C_{\mathrm{CF}_{3} \mathrm{COOH}}=1.4 \times 10^{-3} \div 5.2 \mathrm{M}\right)$ and the corresponding titration curve; $(b)$ The plots of $\log I$ vs $H_{0}$

for $\mathrm{ImC}_{60}$ solutions in $\mathrm{CF}_{3} \mathrm{COOH}-\mathrm{CH}_{2} \mathrm{Cl}_{2}$.

Fig. S2. The plots of $\log I$ vs $\mathrm{H}_{0}$ for $\mathrm{Py}_{3} \mathrm{C}_{60}$ solutions in $\mathrm{CF}_{3} \mathrm{COOH}-\mathrm{CH}_{2} \mathrm{Cl}_{2}$ in the first $(a)$ and second $(b)$ stage.

Fig. S3. The plots of $\log I$ vs $\mathrm{H}_{0}$ for $\mathrm{Py}_{2} \mathrm{C}_{70}$ solutions in $\mathrm{CF}_{3} \mathrm{COOH}-\mathrm{CH}_{2} \mathrm{Cl}_{2}$ in the first $(a)$ and second $(b)$ stage.

Table S2. The total energy in the gas phase and $\mathrm{CH}_{2} \mathrm{Cl}_{2}$ for the $\mathrm{N}$ protonated form of $\mathrm{PyC}_{60} / \mathrm{ImC}_{60}$

Fig. S4. The selected bond lengths $(\AA)$ of ImC60 and PyC60 in the neutral and N monoprotonated forms.

Fig. S5. The selected bond lengths $(\AA)$ of $\mathrm{Py}_{3} \mathrm{C}_{60}$ and $\mathrm{Py}_{2} \mathrm{C}_{70}$ in the neutral and $\mathrm{N}$ protonated forms.

Scheme S1. The thermodynamic cycle of the deprotonation of the fullero[60]pyrrolidine containing pyridyl substituent

Fig. S6. The direction of the dipole moment vector and its value for substituted fullerenes and their singly $\mathrm{N}$ protonated forms.

Fig. S7. The plot of the dipole moment $v s$ of thermodynamic constants ( $\mathrm{p} K$ and $\mathrm{p} K_{\mathrm{BH}+}$ ) for substituted fullerenes.

Fig. S8. The ${ }^{1} \mathrm{H}$ NMR spectrum of $\mathrm{ImC}_{60}$ (black) and its $\mathrm{N}$ protonated form (blue) in $\mathrm{CDCl}_{3} \ldots \mathrm{S} 9$ Fig. S9. The ${ }^{1} \mathrm{H}$ NMR spectrum of $\mathrm{Py}_{3} \mathrm{C}_{60}$ (black) and its $\mathrm{N}$ protonated form (blue) in $\mathrm{CDCl}_{3}$. . S9 Fig. S10. The ${ }^{1} \mathrm{H}$ NMR spectrum of $\mathrm{Py}_{2} \mathrm{C}_{70}$ (black) and its $\mathrm{N}$ protonated form (blue) in $\mathrm{CDCl}_{3}$.

Coordinate of the optimized structure in gas phase. S10 
Synthesis of 1'-N-methyl-2'-(pyridin-4-yl)pyrrolidino[3',4':1,2][60]fullerene, $\mathrm{PyC}_{60}$

A solution of $100 \mathrm{mg}(0.14 \mathrm{mmol})$ of $\mathrm{C}_{60}, 80 \mathrm{mg}(0.70 \mathrm{mmol})$ of pyridine-4-carboxaldehyde and $43 \mathrm{mg}$ $(0.48 \mathrm{mmol})$ of $\mathrm{N}$-methylglycine in $90 \mathrm{ml}$ of toluene was stirred at reflux temperature for $2 \mathrm{~h}$. Then the solvent was removed in vacuum. The formation of $\mathrm{PyC}_{60}$ was monitored by TLC and by the appearance of absorption with $\lambda_{\max }=433 \mathrm{~nm}$ in UV-vis spectrum. The reaction mixture was purified by column chromatography on $\mathrm{Al}_{2} \mathrm{O}_{3}$ (elution with the mixture of toluene- ethyl acetate (20:1)). The yield is $64 \mathrm{mg}$ $(52 \%)$.

Synthesis of 1'-N-methyl-2'-(1H-imidazol-1-yl)-phenylpyrrolidino[3',4':1,2][60]fullerene, ImC 60 A solution of $100 \mathrm{mg}(0.14 \mathrm{mmol})$ of $\mathrm{C}_{60}, 120 \mathrm{mg}(0.70 \mathrm{mmol})$ of 4-(1H-imidazol-1-yl) benzaldehyde and $40 \mathrm{mg}(0.45 \mathrm{mmol})$ of $\mathrm{N}$-methylglycine in $90 \mathrm{ml}$ of toluene was stirred at reflux temperature for $2 \mathrm{~h}$. Then the solvent was removed in vacuum. The reaction mixture was purified over the $\mathrm{Al}_{2} \mathrm{O}_{3}$ column using toluene (for the first zone containing the $\mathrm{C}_{60}$ residue), methanol (for the second zone containing the 4(1H-imidazol-1-yl) benzaldehyde residue) and toluene-ethyl acetate (4:1) (for the third zone containing $\left.\mathrm{ImC}_{60}\right)$ as eluent. The yield is $65 \mathrm{mg}(51 \%)$.

Synthesis of 2'-(Pyridin-4-yl)-5'-(pyridin-2-yl)-1'-(pyridin-2-ylmethyl)-

pyrrolidino[3', $\left.4^{\prime}: 1,2\right][60]$ fullerene, $\mathrm{Py}_{3} \mathrm{C}_{60}$

Pyridine-4-carboxyaldehyde (19 mg, $0.18 \mathrm{mmol})$ and di-(2-picolyl)amine (30 mg, $0.15 \mathrm{mmol})$ dissolved in dichlorobenzene $(3 \mathrm{ml})$ were added to the dichlorobenzene solution of $\mathrm{C}_{60}(100 \mathrm{mg}, 0.14 \mathrm{mmol})$. The reaction mixture was heated at $180^{\circ} \mathrm{C}$ for $10 \mathrm{~min}$. Then the solvent was removed by the vacuum distillation. The reaction mixture was purified over the $\mathrm{Al}_{2} \mathrm{O}_{3}$ column using toluene (for the first zone containing the $\mathrm{C}_{60}$ residue), toluene- methanol (40:1) (for the third zone containing $\mathrm{Py}_{3} \mathrm{C}_{60}$ ) as eluent. The yield is $78 \mathrm{mg}(55 \%)$.

Synthesis of 2',5'-di-(pyridin-2-yl) pyrrolidino[3',4':1,2][70]fullerene, $\mathrm{Py}_{2} \mathrm{C}_{70}$

Pyridine-2-carboxyaldehyde (15 mg, $0.14 \mathrm{mmol})$ and 2-picolylamine $(15 \mathrm{mg}, 0.14 \mathrm{mmol})$ dissolved in dichlorobenzene $(3 \mathrm{ml})$ were added to the dichlorobenzene solution of $\mathrm{C}_{70}(100 \mathrm{mg}, 0.12 \mathrm{mmol})$. The reaction mixture was heated at $180^{\circ} \mathrm{C}$ for $15 \mathrm{~min}$. Then the solvent was removed by vacuum distillation. The residue was dissolved with toluene and adsorbed on silica gel. The first zone with toluene as an eluent is the $\mathrm{C}_{70}$ residue, and the second zone with toluene:methanol (20:1) as an eluent is $\mathrm{Py}_{2} \mathrm{C}_{70}$. The yield is $45 \mathrm{mg}(45 \%)$. 
Table S1. The UV-vis spectra of substituted fullero[60]/[70]pyrrolidines in toluene and $\mathrm{CH}_{2} \mathrm{Cl}_{2}$.

\begin{tabular}{c|c|c}
\hline \multirow{2}{*}{ Fullerene } & \multicolumn{2}{|c}{ UV-vis spectra } \\
\cline { 2 - 3 } & toluene & $\mathrm{CH}_{2} \mathrm{Cl}_{2}$ \\
\hline $\mathrm{PyC}_{60}$ & $312(4.54), 433(3.56)$ & $309(4.62), 431(3.65)$ \\
\hline $\mathrm{ImC}_{60}$ & $311(4.45), 434(3.54)$ & $308(4.55), 431(3.56)$ \\
\hline $\mathrm{Py}_{3} \mathrm{C}_{60}$ & $313(4.56), 433(3.58)$ & $310(4.66), 430(3.62)$ \\
\hline $\mathrm{Py}_{2} \mathrm{C}_{70}$ & $398(4.91), 464(4.23)$ & $394(4.20), 462(4.14)$ \\
\hline
\end{tabular}

Fig. S1. (a) The UV-vis spectra of $\mathrm{ImC}_{60}$ in $\mathrm{CF}_{3} \mathrm{COOH}-\mathrm{CH}_{2} \mathrm{Cl}_{2}$ obtained during titration $\left(C_{\mathrm{CF}_{3} \mathrm{COOH}}=1.4 \times 10^{-3} \div 5.2 \mathrm{M}\right)$ and the corresponding titration curve; $(b)$ The plots of $\log I$ vs $H_{0}$ for $\mathrm{ImC}_{60}$ solutions in $\mathrm{CF}_{3} \mathrm{COOH}-\mathrm{CH}_{2} \mathrm{Cl}_{2}$.
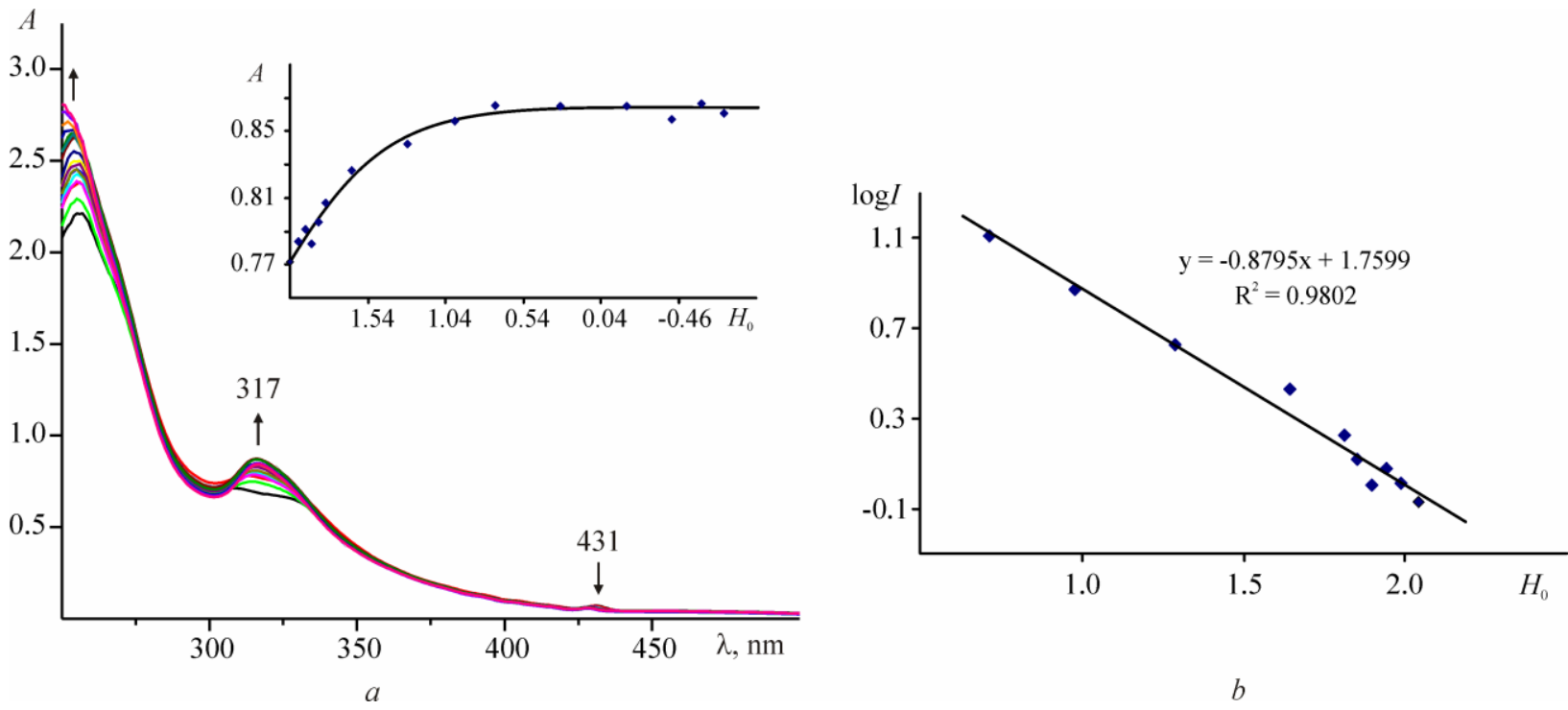

Fig. S2. The plots of $\log I$ vs $\mathrm{H}_{0}$ for $\mathrm{Py}_{3} \mathrm{C}_{60}$ solutions in $\mathrm{CF}_{3} \mathrm{COOH}-\mathrm{CH}_{2} \mathrm{Cl}_{2}$ in the first $(a)$ and second $(b)$ stage.
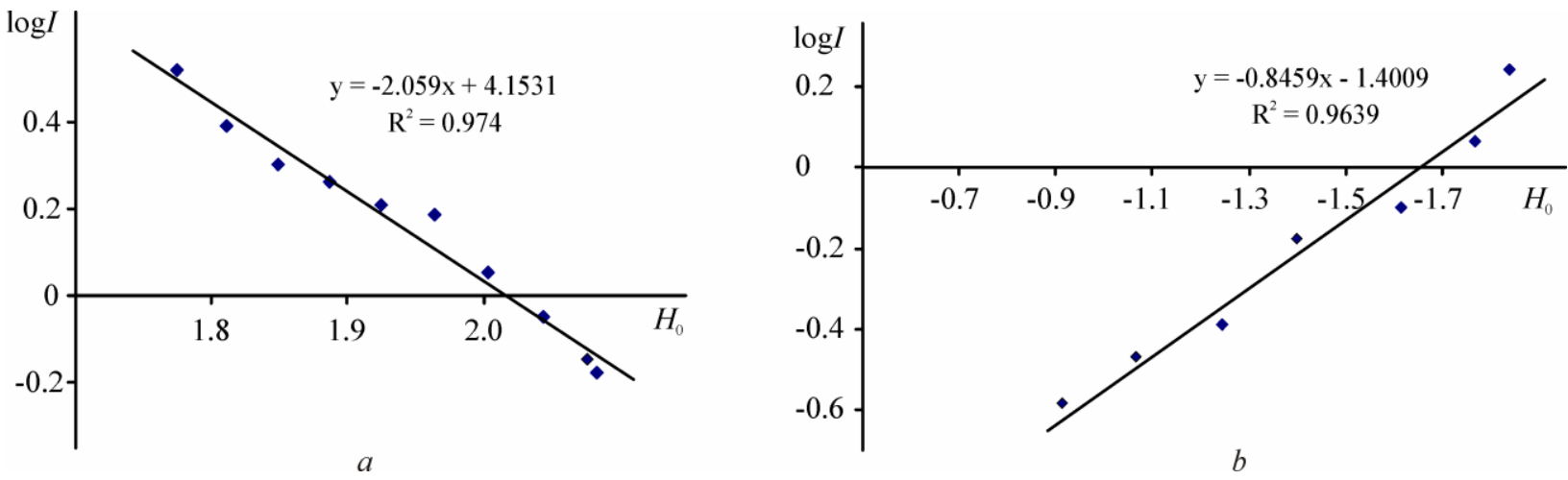
Fig. S3. The plots of $\log I$ vs $\mathrm{H}_{0}$ for $\mathrm{Py}_{2} \mathrm{C}_{70}$ solutions in $\mathrm{CF}_{3} \mathrm{COOH}-\mathrm{CH}_{2} \mathrm{Cl}_{2}$ in the first $(a)$ and second (b) stage.
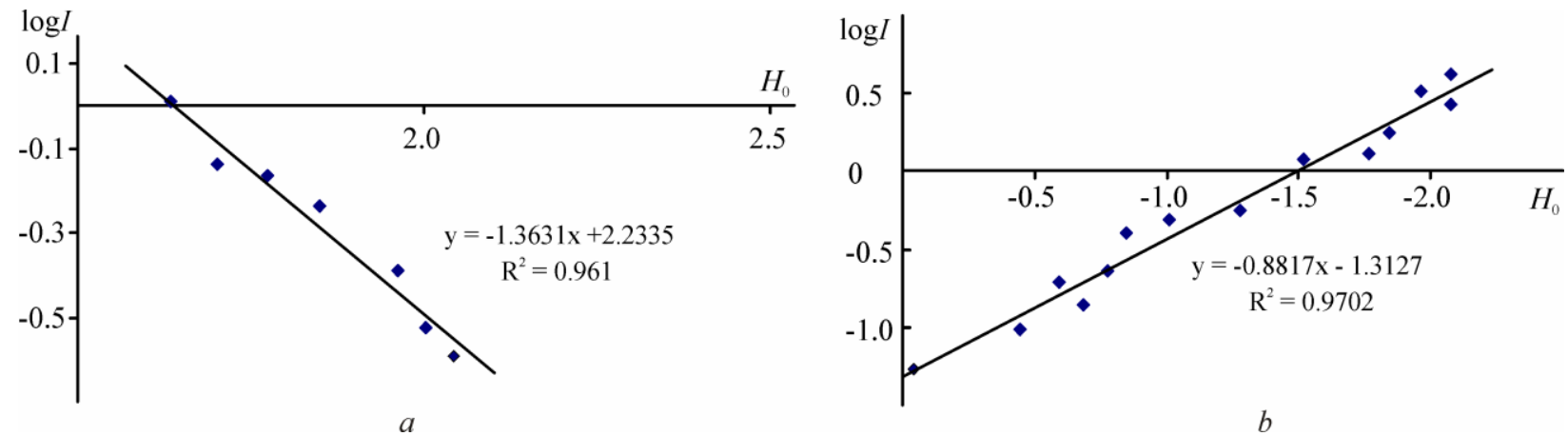

Table S2. The total energy in the gas phase and $\mathrm{CH}_{2} \mathrm{Cl}_{2}$ for the $\mathrm{N}$ protonated form of $\mathrm{PyC}_{60} / \mathrm{ImC}_{60}$.

\begin{tabular}{lcc}
\hline Isomer & $\begin{array}{c}\text { Total energy in gas } \\
\text { phase, a.u. }\end{array}$ & $\begin{array}{c}\text { Total energy in } \mathrm{CH}_{2} \mathrm{Cl}_{2}, \\
\text { a.u. }\end{array}$ \\
\hline $\mathrm{PyC}_{60} \mathrm{H}^{+}$ & -2707.42252 & -2707.48990 \\
$\mathrm{PyH}^{+} \mathrm{C}_{60}$ & -2707.42481 & -2707.49423 \\
$\mathrm{ImC}_{60} \mathrm{H}^{+}$ & -2916.45775 & -2916.52790 \\
$\mathrm{ImH}^{+} \mathrm{C}_{60}$ & -2916.46483 & -2916.53554 \\
$\mathrm{Py}_{3} \mathrm{H}^{+} \mathrm{C}_{60}$ & -3201.72832 & -3201.78946 \\
$\mathrm{Py}_{3} \mathrm{C}_{60} \mathrm{H}^{+}$ & -3201.73902 & -3201.79222 \\
$\mathrm{Py}_{3} \mathrm{H}^{+}{ }_{2-\mathrm{Py}} \mathrm{C}_{60} *$ & -3201.74127 & -3201.80353 \\
$\mathrm{Py}_{3} \mathrm{H}^{+}{ }_{3-\mathrm{Py}} \mathrm{C}_{60}{ }^{* *}$ & -3201.72359 & -3201.78563 \\
$\mathrm{Py}_{2} \mathrm{H}^{+} \mathrm{C}_{70}$ & -3296.48783 & -3296.54482 \\
$\mathrm{Py}_{2} \mathrm{C}_{70} \mathrm{H}^{+}$ & -3296.48218 & -3296.53916
\end{tabular}

*The proton is located at 2-pyridyl ring (Fig. 1 main text) **The proton is located at 3-pyridyl ring (Fig. 1 main text). Energy was calculated by the DFT B3lyp/6-311G(d,p) method. 
Fig. S4. The selected bond lengths $(\AA)$ of $\mathrm{ImC}_{60}$ and $\mathrm{PyC}_{60}$ in the neutral and $\mathrm{N}$ monoprotonated forms.

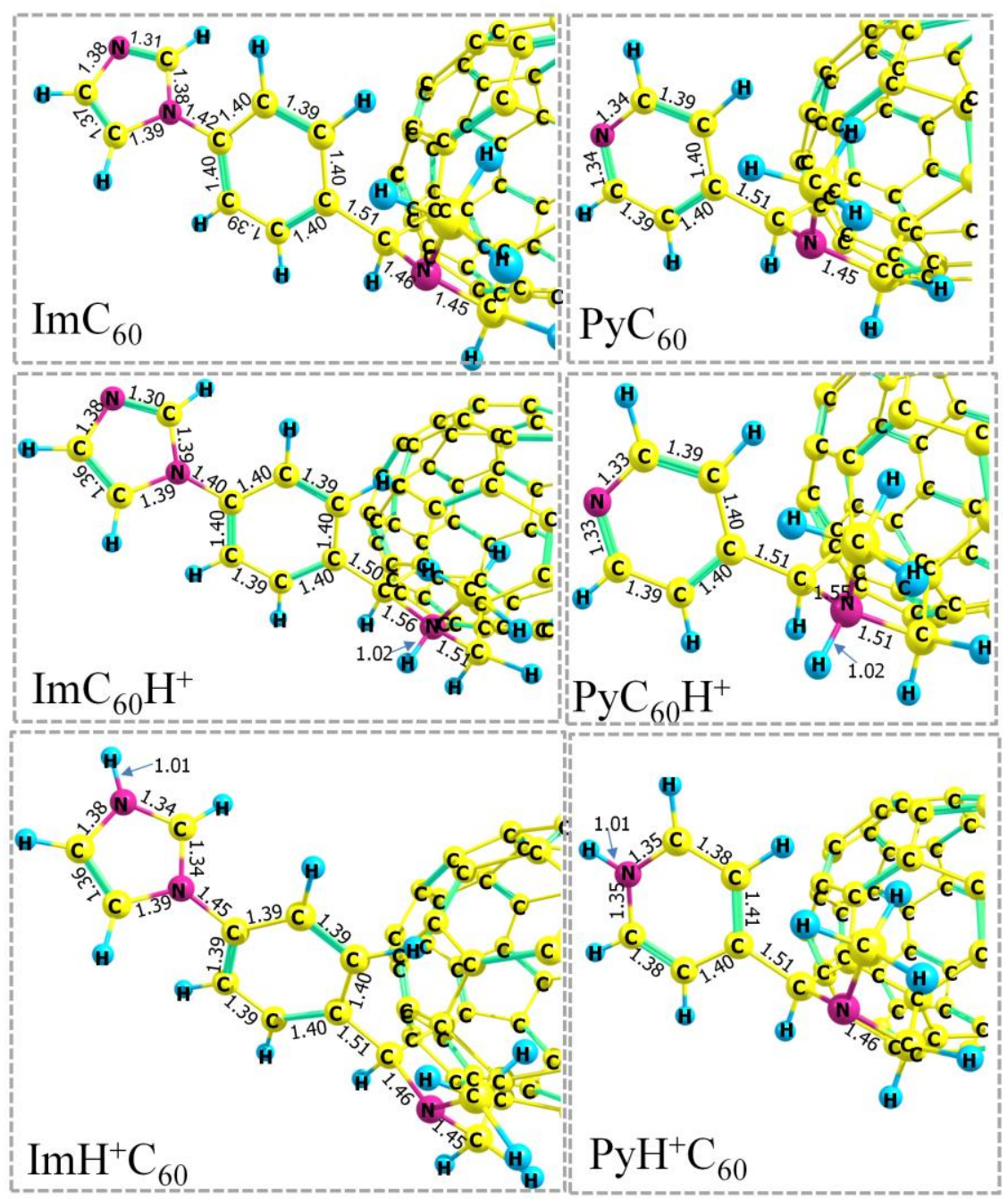


Fig. S5. The selected bond lengths $(\AA)$ of $\mathrm{Py}_{3} \mathrm{C}_{60}$ and $\mathrm{Py}_{2} \mathrm{C}_{70}$ in the neutral and $\mathrm{N}$ protonated forms.

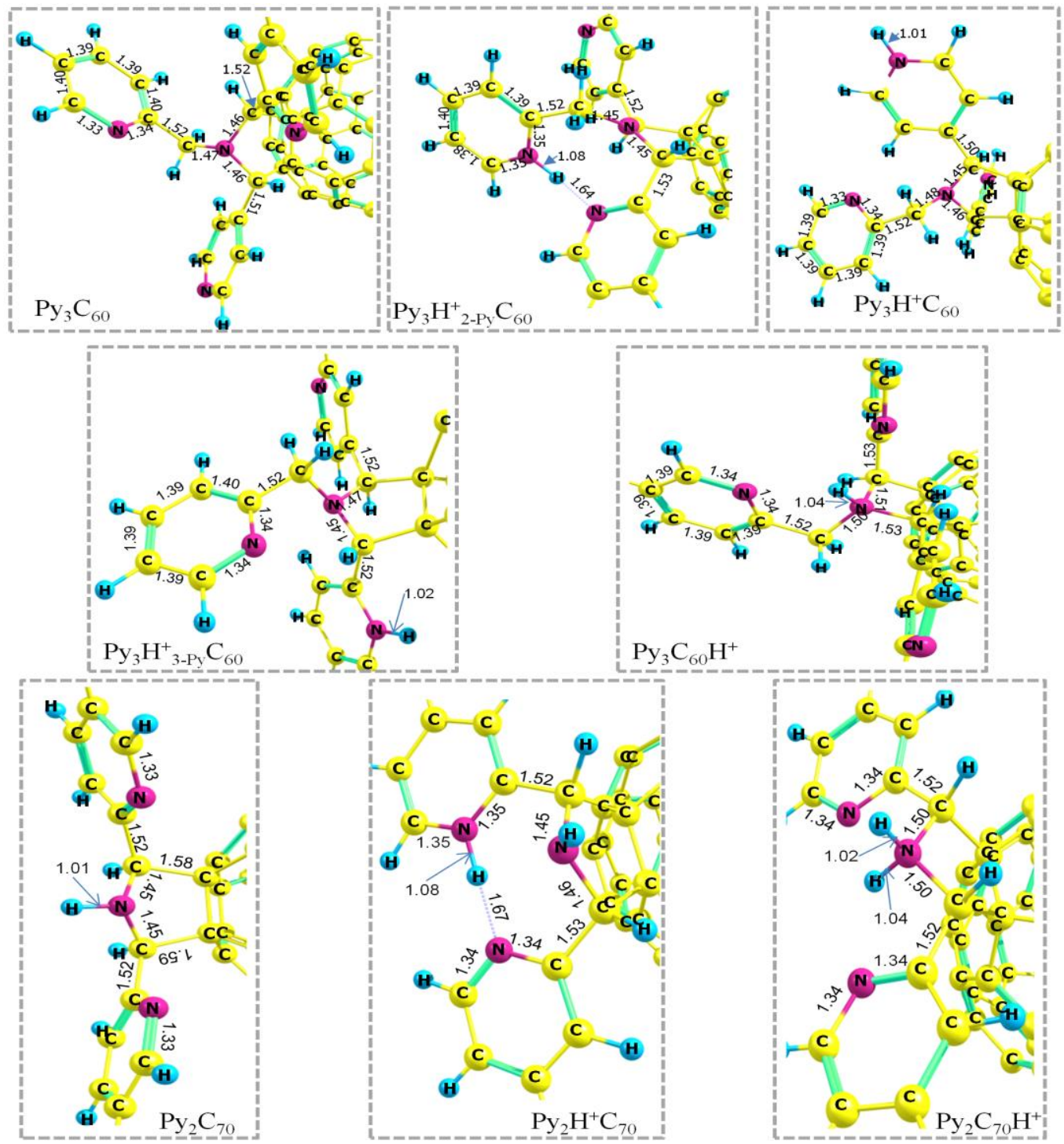

Scheme S1. The thermodynamic cycle of the deprotonation of the conjugate acid for a fullero[60]pyrrolidine base containing pyridyl substituent.

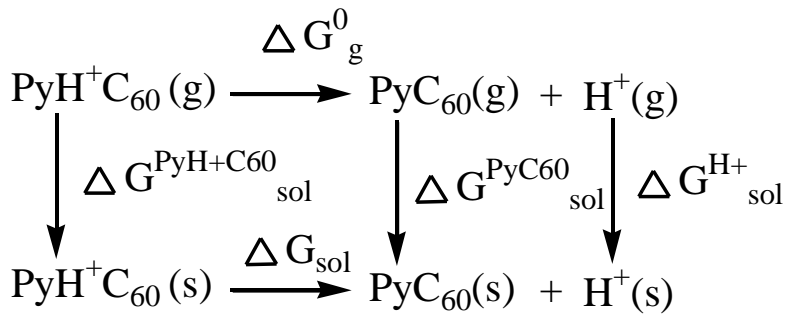


Fig. S6. The direction of the dipole moment vector and its value for substituted fullerenes and their singly $\mathrm{N}$ protonated forms.

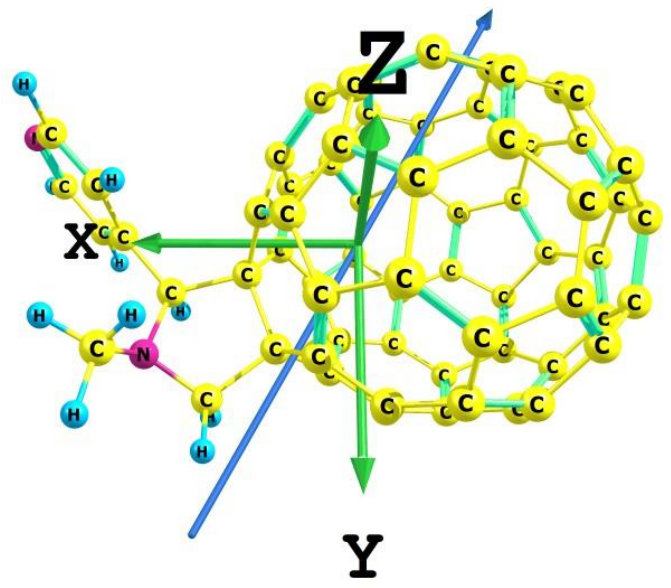

$\mathrm{PyC}_{60}$

Dipole moment (Debye):

$\mathrm{X}=2.5487 \mathrm{Y}=4.6968 \mathrm{Z}=-0.3326$

Tot $=5.4182$

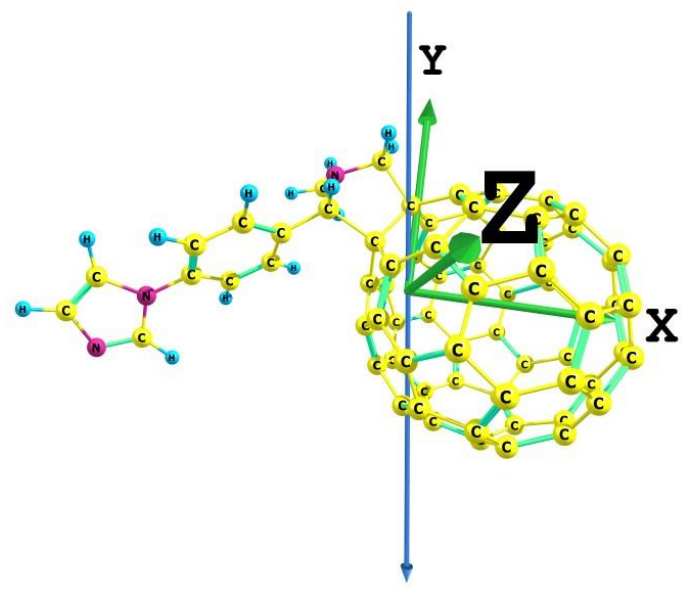

$\mathrm{ImC}_{60}$

Dipole moment (Debye):

$\mathrm{X}=-1.0350 \mathrm{Y}=7.3092 \mathrm{Z}=1.4071$

Tot $=7.5150$

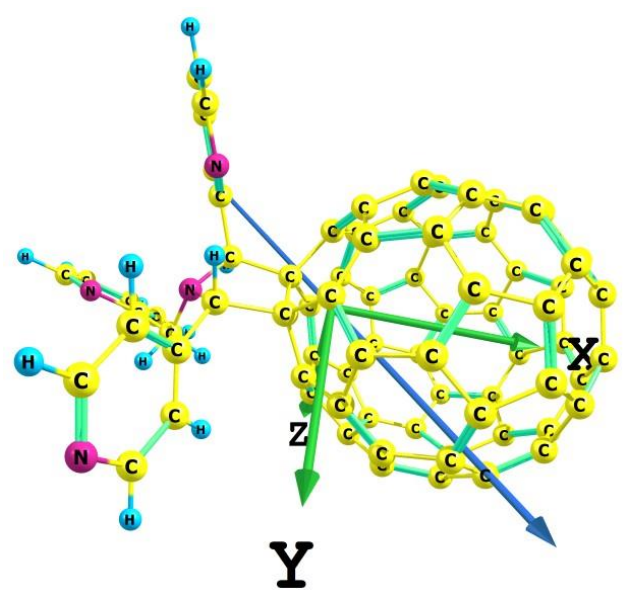

$\mathrm{Py}_{3} \mathrm{C}_{60}$

Dipole moment (Debye):

$\mathrm{X}=-5.3030 \mathrm{Y}=-7.6025 \mathrm{Z}=2.8674$

$\mathrm{Tot}=9.7026$

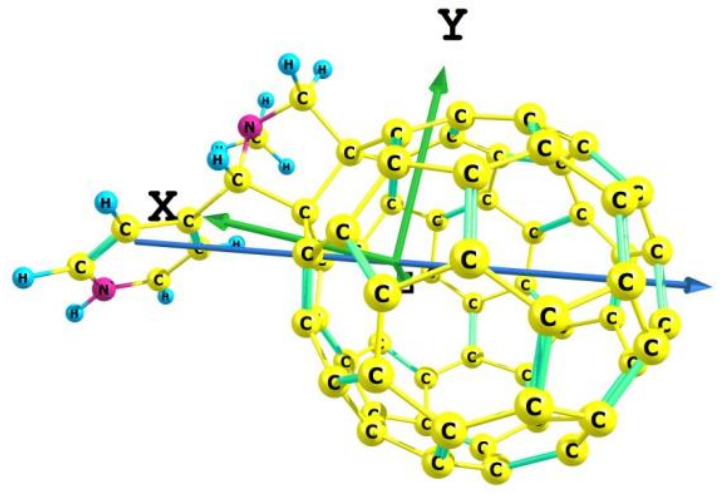

$\mathrm{PyH}^{+} \mathrm{C}_{60}$

Dipole moment (Debye):

$\mathrm{X}=33.9262 \mathrm{Y}=-4.5010 \mathrm{Z}=0.1148$

Tot $=34.2237$

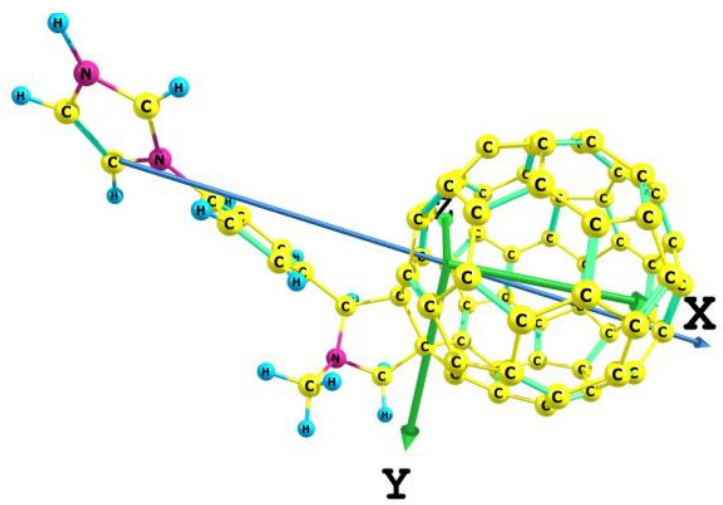

$\operatorname{ImH}^{+} \mathrm{C}_{60}$

Dipole moment (Debye):

$\mathrm{X}=-43.1572 \mathrm{Y}=-4.4531 \mathrm{Z}=-1.0677$

$\mathrm{Tot}=43.3994$

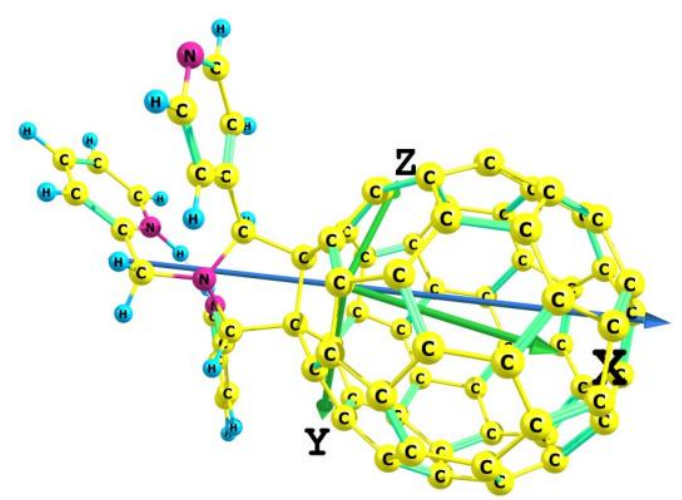

$\mathrm{Py}_{3} \mathrm{H}^{+}{ }_{2-\mathrm{Py}} \mathrm{C}_{60}$

Dipole moment (Debye):

$\mathrm{X}=-32.3741 \mathrm{Y}=9.1140 \mathrm{Z}=-0.8057$

Tot $=33.6422$ 


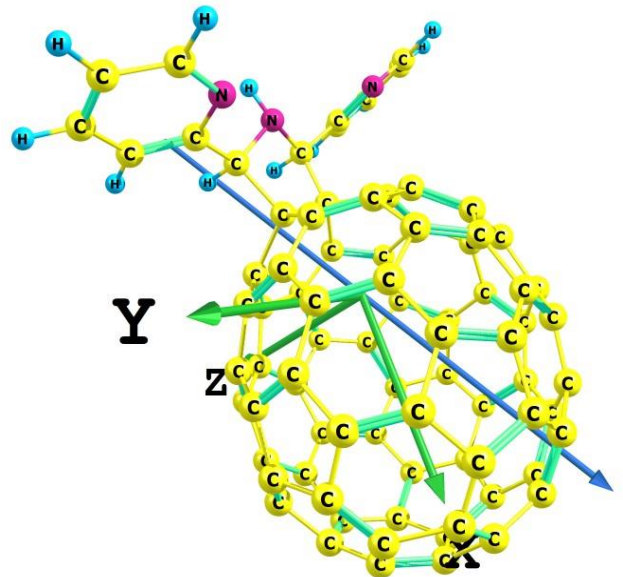

$\mathrm{Py}_{2} \mathrm{C}_{70}$

Dipole moment (Debye):

$\mathrm{X}=-9.2824 \mathrm{Y}=2.4920 \mathrm{Z}=6.3354$

Tot $=11.5113$

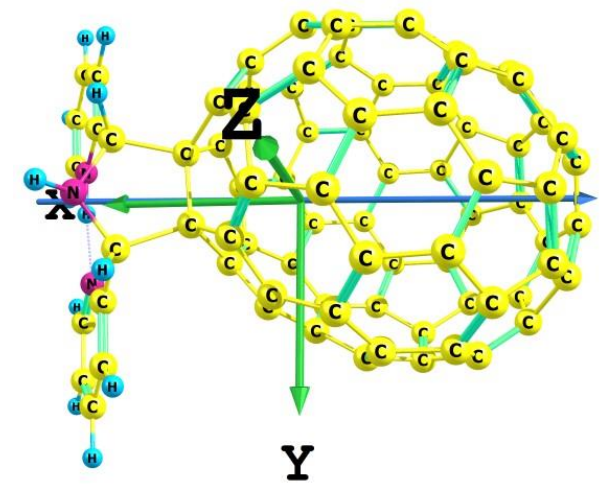

$\mathrm{Py}_{2} \mathrm{H}^{+} \mathrm{C}_{70}$

Dipole moment (Debye):

$\mathrm{X}=30.5168 \mathrm{Y}=-0.2836 \mathrm{Z}=1.8534$

Tot $=30.5744$

Fig. S7. The plot of the dipole moment $v s$ of thermodynamic constants ( $\mathrm{p} K$ and $\mathrm{p} K_{\mathrm{BH}+}$ ) for substituted fullerenes.

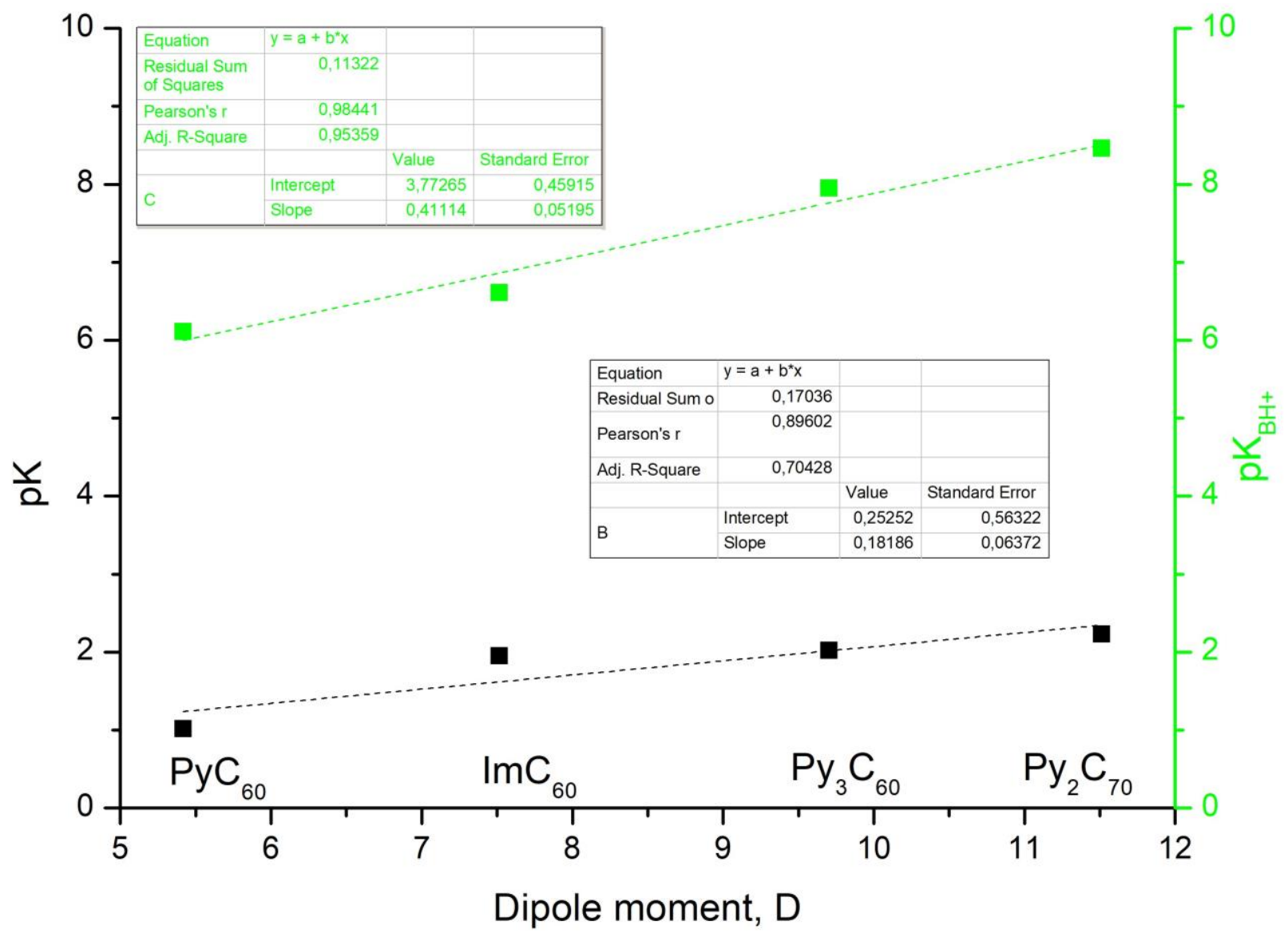


Fig. S8. The ${ }^{1} \mathrm{H}$ NMR spectrum of $\mathrm{ImC}_{60}$ (black) and its $\mathrm{N}$ protonated form (blue) in $\mathrm{CDCl}_{3}$.

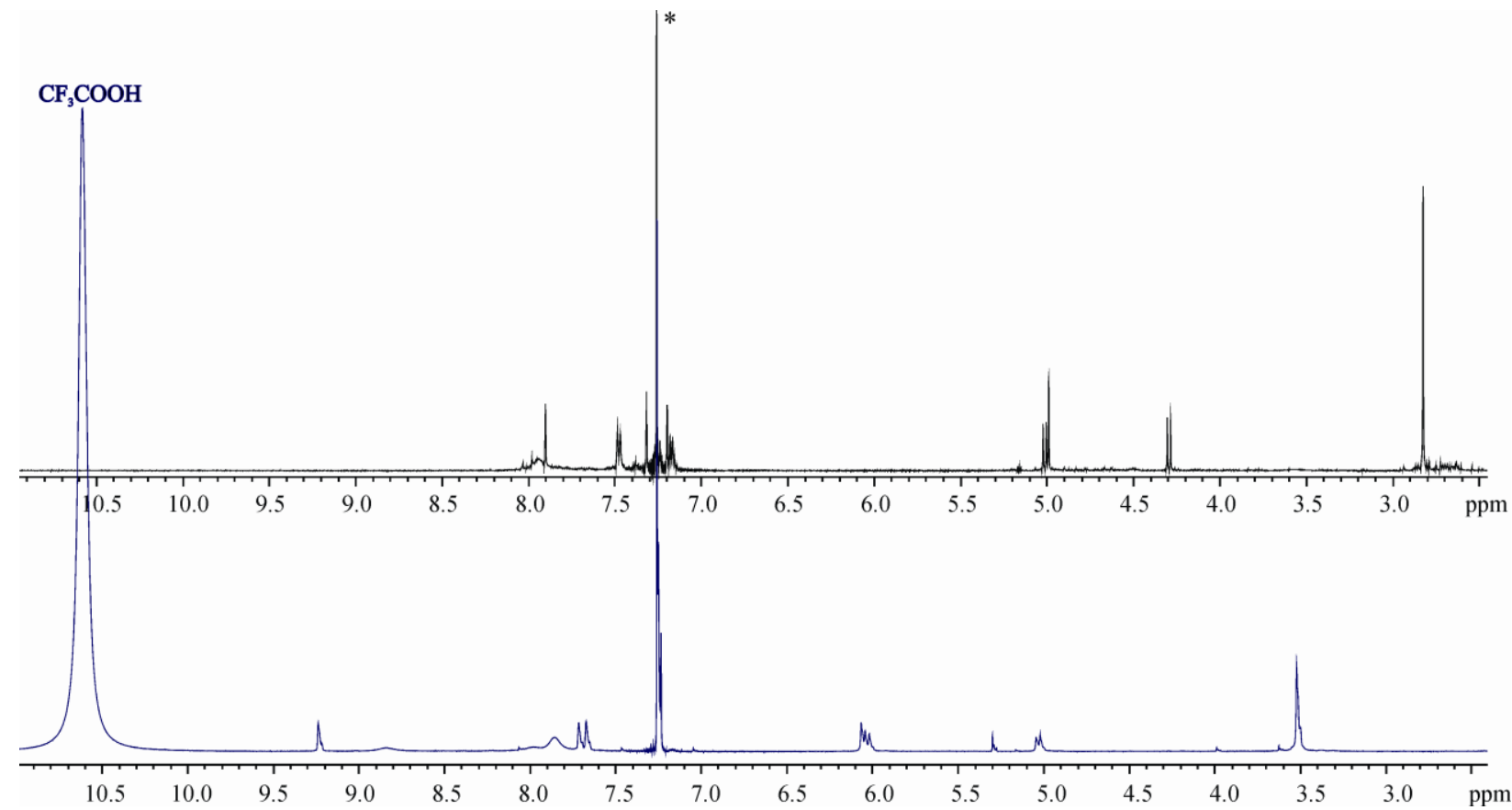

Fig. S9. The ${ }^{1} \mathrm{H}$ NMR spectrum of $\mathrm{Py}_{3} \mathrm{C}_{60}$ (black) and its $\mathrm{N}$ protonated form (blue) in $\mathrm{CDCl}_{3}$.

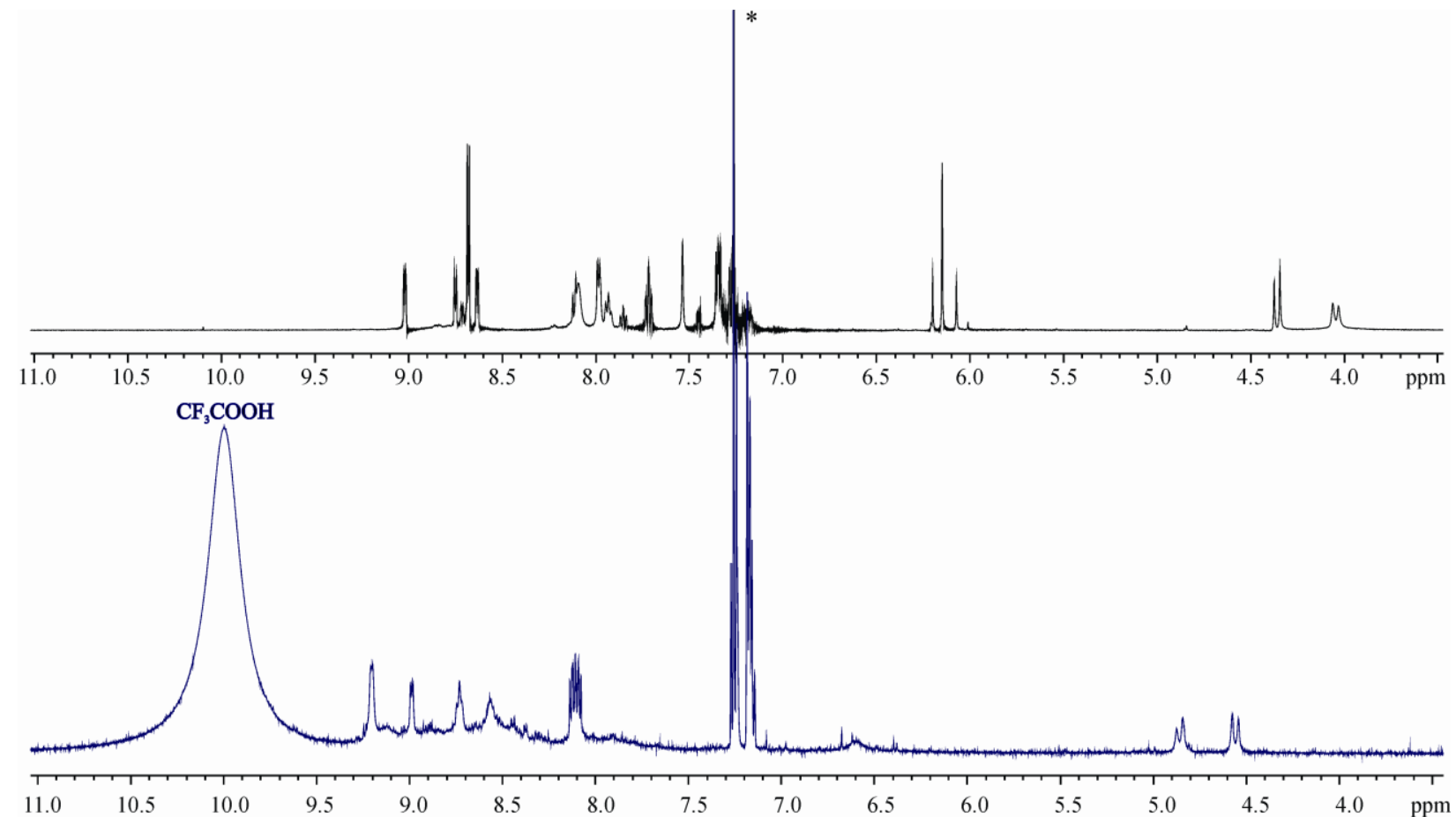


Fig. S10. The ${ }^{1} \mathrm{H}$ NMR spectrum of $\mathrm{Py}_{2} \mathrm{C}_{70}$ (black) and its $\mathrm{N}$ protonated form (blue) in $\mathrm{CDCl}_{3}$.

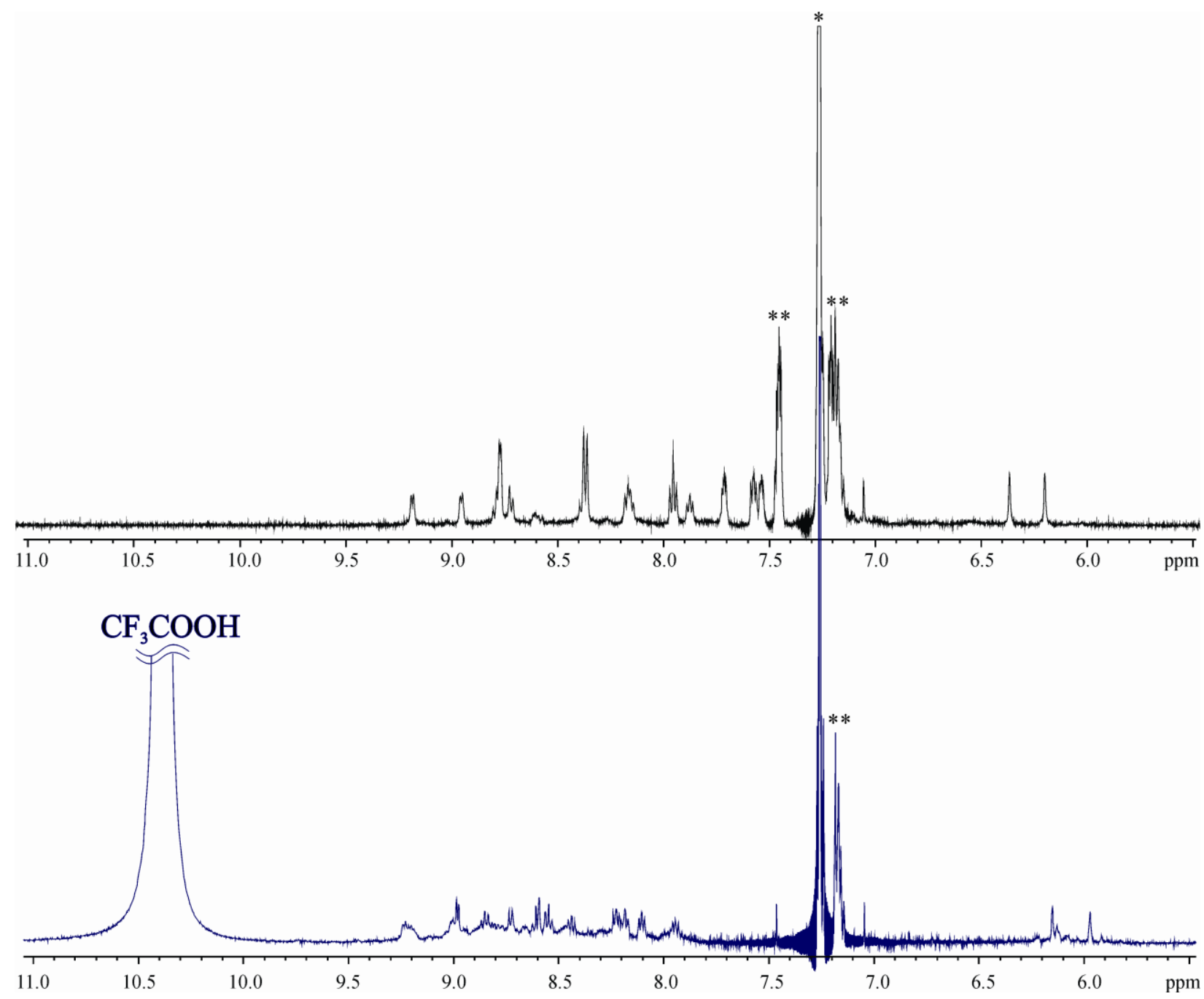

Coordinate of the optimized structure in gas phase.

PyC60/b31yp/6-311G(d,p)

$\begin{array}{lrrr}\mathrm{H} & 3.284691000 & 3.961816000 & -0.027120000 \\ \mathrm{C} & 0.162340000 & -3.324026000 & -0.636099000 \\ \mathrm{C} & -0.908434000 & -3.238502000 & 1.578978000 \\ \mathrm{C} & 0.280150000 & -3.249002000 & 0.747410000 \\ \mathrm{C} & -1.699115000 & -1.528021000 & 3.165413000 \\ \mathrm{C} & -1.435101000 & -0.142841000 & 3.513728000 \\ \mathrm{C} & -2.590841000 & 0.647480000 & 3.128035000 \\ \mathrm{C} & -2.169279000 & -3.297482000 & 0.989790000 \\ \mathrm{C} & -2.292732000 & -3.373505000 & -0.455545000 \\ \mathrm{C} & -3.010154000 & -1.591171000 & 2.554342000 \\ \mathrm{C} & -3.241536000 & -2.457124000 & 1.487609000 \\ \mathrm{C} & -4.028791000 & -2.014041000 & 0.351743000 \\ \mathrm{C} & -3.561354000 & -0.246058000 & 2.531054000 \\ \mathrm{C} & -4.320992000 & 0.175673000 & 1.442241000 \\ \mathrm{C} & -4.556877000 & -0.725666000 & 0.329592000 \\ \mathrm{C} & -3.441149000 & -2.579539000 & -0.848946000\end{array}$




\begin{tabular}{|c|c|c|c|}
\hline & -3.399580000 & -1.829221000 & \\
\hline & & & \\
\hline & -3.951822000 & -0.484253000 & \\
\hline & -4.141168000 & 1.511627000 & 907642000 \\
\hline & -4.264809000 & & \\
\hline & -2.210485000 & -1.840604000 & \\
\hline & -2.021301000 & -0.501120000 & \\
\hline & -3.102971000 & 09000 & \\
\hline & -1.149826000 & -3.387962000 & \\
\hline & 102000 & -2.60 & \\
\hline 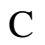 & -0.66 & -2.3 & 2.6 \\
\hline & -3.451393000 & 2.226012000 & \\
\hline$C$ & -2.85 & 000 & \\
\hline$C$ & -2.418043000 & & \\
\hline$C$ & -3.2 & & \\
\hline$C$ & -2.36 & & \\
\hline 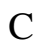 & 0072000 & 0000 & -0.7 \\
\hline 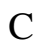 & -0.47 & & \\
\hline & $0.412 \varepsilon$ & -0.7 & \\
\hline & 1.9 & -1.6 & \\
\hline$C$ & 2.14 & -0.3 & \\
\hline & 1.39 & & \\
\hline C & 0.82 & & \\
\hline$c$ & 1.05 & 2.3 & \\
\hline$C$ & 2.70 & 0.6 & \\
\hline & 2.0900 & & \\
\hline $\mathrm{C}$ & 2.35 & -0.1 & \\
\hline & 1.77 & & \\
\hline $\mathrm{C}$ & 1.213 & & \\
\hline & 1.259 & 2.46 & \\
\hline $\mathrm{C}$ & 2.053 & -1.5 & \\
\hline $\mathrm{C}$ & -0.026 & & \\
\hline $\mathrm{C}$ & 0.09 & & \\
\hline $\mathrm{C}$ & 1.24 & -2.3 & \\
\hline $\mathrm{C}$ & 1.008615000 & -2.500 & -1.4 \\
\hline $\mathrm{C}$ & -1.285 & 3.10 & -1.5 \\
\hline $\mathrm{C}$ & -1.047608000 & & \\
\hline $\mathrm{C}$ & 0.919308000 & $-0.45^{\circ}$ & \\
\hline $\mathrm{C}$ & 0.028289000 & & \\
\hline $\mathrm{C}$ & 0.230174000 & -2.061579000 & -2.61 \\
\hline $\mathrm{C}$ & -1.522056000 & & \\
\hline $\mathrm{C}$ & -1.084109000 & 44000 & 778000 \\
\hline $\mathrm{C}$ & 0.667963000 & -1.792228000 & 426000 \\
\hline $\mathrm{C}$ & -0.737384000 & 0.023370000 & -3.494555000 \\
\hline $\mathrm{C}$ & -0.154706000 & 0.379901000 & \\
\hline $\mathrm{C}$ & 4.266087000 & & -0.64039100 \\
\hline $\mathrm{N}$ & 4.535797000 & 2.265861000 & -0.13626700 \\
\hline $\mathrm{C}$ & & 3.020959000 & -0.56876200 \\
\hline 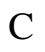 & 4.908035000 & 2.437121000 & 1.26896000 \\
\hline
\end{tabular}




$\begin{array}{lrrr}\mathrm{H} & 4.302050000 & 1.020268000 & -1.730599000 \\ \mathrm{H} & 3.473438000 & 3.248086000 & -1.634928000 \\ \mathrm{H} & 4.167966000 & 2.071991000 & 1.993999000 \\ \mathrm{H} & 5.061311000 & 3.502735000 & 1.449049000 \\ \mathrm{C} & 5.271572000 & -0.151400000 & -0.285570000 \\ \mathrm{C} & 6.113969000 & -0.638862000 & -1.288198000 \\ \mathrm{C} & 7.051021000 & -1.623047000 & -0.985326000 \\ \mathrm{~N} & 7.203912000 & -2.148082000 & 0.232566000 \\ \mathrm{C} & 6.398904000 & -1.683457000 & 1.191697000 \\ \mathrm{C} & 5.430613000 & -0.702573000 & 0.989792000 \\ \mathrm{H} & 6.044089000 & -0.255459000 & -2.299970000 \\ \mathrm{H} & 7.708849000 & -2.007444000 & -1.759906000 \\ \mathrm{H} & 6.530672000 & -2.118829000 & 2.178352000 \\ \mathrm{H} & 5.856253000 & 1.934320000 & 1.458613000 \\ \mathrm{H} & 4.814409000 & -0.390516000 & 1.821560000\end{array}$

$\begin{array}{lrrr}\mathrm{PyH}^{+} \mathrm{C}_{60} / \mathrm{b} 31 \mathrm{yp} / 6-311 \mathrm{G}(\mathrm{d}, \mathrm{p}) & \\ \mathrm{H} & 3.218182000 & 4.035348000 & -0.102999000 \\ \mathrm{C} & 0.207235000 & -3.306982000 & -0.650200000 \\ \mathrm{C} & -0.832149000 & -3.238541000 & 1.580937000 \\ \mathrm{C} & 0.343425000 & -3.230677000 & 0.731502000 \\ \mathrm{C} & -1.626696000 & -1.537235000 & 3.174533000 \\ \mathrm{C} & -1.380057000 & -0.147324000 & 3.520051000 \\ \mathrm{C} & -2.553820000 & 0.623445000 & 3.151489000 \\ \mathrm{C} & -2.099304000 & -3.315240000 & 1.008347000 \\ \mathrm{C} & -2.241995000 & -3.393810000 & -0.435031000 \\ \mathrm{C} & -2.945478000 & -1.621841000 & 2.584102000 \\ \mathrm{C} & -3.177489000 & -2.491474000 & 1.520868000 \\ \mathrm{C} & -3.987969000 & -2.062224000 & 0.396577000 \\ \mathrm{C} & -3.517972000 & -0.285762000 & 2.568786000 \\ \mathrm{C} & -4.298589000 & 0.123330000 & 1.490634000 \\ \mathrm{C} & -4.535183000 & -0.782158000 & 0.381632000 \\ \mathrm{C} & -3.408313000 & -2.618202000 & -0.811963000 \\ \mathrm{C} & -3.395430000 & -1.868028000 & -1.985599000 \\ \mathrm{C} & -4.529529000 & -0.002231000 & -0.842186000 \\ \mathrm{C} & -3.969206000 & -0.532200000 & -2.001981000 \\ \mathrm{C} & -4.147597000 & 1.461596000 & 0.953173000 \\ \mathrm{C} & -4.290654000 & 1.384030000 & -0.489719000 \\ \mathrm{C} & -2.217719000 & -1.860697000 & -2.827309000 \\ \mathrm{C} & -2.057620000 & -0.518385000 & -3.360043000 \\ \mathrm{C} & -3.145777000 & 0.299716000 & -2.854394000 \\ \mathrm{C} & -1.111467000 & -3.393025000 & -1.248762000 \\ \mathrm{C} & -1.098708000 & -2.609379000 & -2.471157000 \\ \mathrm{C} & -0.590753000 & -2.331001000 & 2.688041000 \\ \mathrm{C} & -3.502583000 & 2.185885000 & -1.311058000 \\ \mathrm{C} & -2.916168000 & 1.631774000 & -2.518781000 \\ \mathrm{C} & -2.408549000 & 1.910251000 & 2.638402000 \\ \mathrm{C} & -3.223852000 & 2.338422000 & 1.515734000 \\ \mathrm{C} & -2.400690000 & 3.170126000 & 0.658437000\end{array}$




\begin{tabular}{|c|c|c|c|}
\hline & -2.537015000 & 3.094436000 & \\
\hline & -0.542937000 & & \\
\hline & 0.381366000 & -0.746923000 & \\
\hline & 1.947964000 & -1.581358000 & -0.94 \\
\hline & 2.134612000 & -0.265071000 & \\
\hline & 1.351651000 & 0.151133000 & \\
\hline & 0.771935000 & 1.510550000 & \\
\hline & 0.997824000 & & \\
\hline$C$ & 2.681254000 & 0.676786000 & \\
\hline & 2.052468000 & 69000 & \\
\hline$C$ & 2.373233000 & -0.133257000 & \\
\hline & 1.793508000 & 0.396 & \\
\hline $\mathrm{C}$ & 1.216326000 & 1.756037000 & \\
\hline$C$ & 1.233004000 & 2.504 & \\
\hline$C$ & 2.090037000 & -1.50 & 000 \\
\hline$C$ & 6000 & 3.10 & \\
\hline C & 0.056282000 & 3.24 & 000 \\
\hline$C$ & 1.3 & -2.3 & \\
\hline 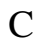 & 92000 & -2.46 & 000 \\
\hline 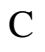 & -1.35 & 3.09 & \\
\hline C & 3715000 & 3.25 & 1000 \\
\hline$C$ & 37000 & -0.42 & \\
\hline $\mathrm{C}$ & 000 & 1.7 & \\
\hline C & 0.226679000 & -2.04 & -2 \\
\hline C & -1.5 & 2.2 & \\
\hline & -1.08 & & \\
\hline 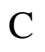 & & -1.7 & \\
\hline & -0.785236000 & 0.026 & 000 \\
\hline$C$ & -0.11 & 0.39 & 000 \\
\hline 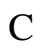 & 4.236582000 & 1.016 & -0.6 \\
\hline $\mathrm{N}$ & 00 & 2.35 & 000 \\
\hline $\mathrm{C}$ & 3.316 & 3.08 & \\
\hline $\mathrm{C}$ & 4.86 & 2.55 & 000 \\
\hline $\mathrm{H}$ & & 1.10 & 000 \\
\hline $\mathrm{H}$ & 3.413860000 & 3.30 & 9000 \\
\hline $\mathrm{H}$ & 000 & 2.16 & \\
\hline $\mathrm{H}$ & & & \\
\hline C & 5.196930000 & -0.07 & 0000 \\
\hline $\mathrm{C}$ & & -0.75 & 0000 \\
\hline $\mathrm{C}$ & 6.742218000 & -1.794327000 & 79000 \\
\hline $\mathrm{N}$ & & -2.167163000 & 0.260322000 \\
\hline $\mathrm{C}$ & 6.260016000 & -1.556110000 & 84000 \\
\hline $\mathrm{C}$ & & -0.51 & 1000 \\
\hline $\mathrm{H}$ & 5.770021000 & -0.460310000 & -2.359521000 \\
\hline $\mathrm{H}$ & 7.300411000 & -2.345378000 & -1.770762000 \\
\hline $\mathrm{H}$ & & -1.934556000 & 2.27263100 \\
\hline $\mathrm{H}$ & 5.851945000 & 2.129449000 & 1.407317000 \\
\hline $\mathrm{H}$ & & -0.056600000 & 1.84223300 \\
\hline & 7.532369000 & -2.936224000 & 0.47118500 \\
\hline
\end{tabular}




\begin{tabular}{|c|c|c|c|}
\hline \multicolumn{4}{|c|}{$\mathrm{PyC}_{60} \mathrm{H}^{+} / \mathrm{b} 31 y p / 6-311 \mathrm{G}(\mathrm{d}, \mathrm{p})$} \\
\hline $\mathrm{H}$ & 3.244727000 & 3.955636000 & \\
\hline C & 0.135567000 & -3.330888000 & \\
\hline & -0.942333000 & -3.247254000 & \\
\hline & 0.248248000 & -3.260414000 & 3000 \\
\hline & -1.731015000 & -1.534514000 & 12000 \\
\hline & -1.461873000 & -0.151182000 & 3.50 \\
\hline & -2.612977000 & 0.645028000 & \\
\hline & -2.200154000 & -3.296 & 4000 \\
\hline & -2.318236000 & -3.3686 & 1000 \\
\hline & -3.039 & & \\
\hline & -3.270 & -2.452 & 2000 \\
\hline & -4.050 & -2.003 & \\
\hline & -3.585 & -0.242 & 000 \\
\hline & -4.33 & 0.18 & \\
\hline $\mathrm{C}$ & -4.57 & -0.712 & 1000 \\
\hline & -3.46 & -2.5 & \\
\hline & -3.412 & -1.8 & 3000 \\
\hline & -4.5 & & \\
\hline 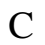 & -3.958 & -0.46 & 2000 \\
\hline & -4.15 & & \\
\hline $\mathrm{C}$ & -4.26 & & 3000 \\
\hline & -2.21 & -1.830 & -2 \\
\hline$C$ & -2.022 & -0.4 & \\
\hline & -3.102 & 0.3 & 000 \\
\hline C & -1.17 & -3.3 & 1000 \\
\hline & -1.12268 & -2.60 & 2000 \\
\hline$C$ & -0.70 & -2.3 & 000 \\
\hline & -3.45 & & 000 \\
\hline$C$ & -2.85 & 1.6 & 5000 \\
\hline & -2.434 & 1.930 & 1000 \\
\hline $\mathrm{C}$ & -3.220 & & .000 \\
\hline & -2.36 & & 2000 \\
\hline$C$ & -2.477 & 3.125 & 7000 \\
\hline $\mathrm{C}$ & -0.474 & & 000 \\
\hline & & -0.770 & 2000 \\
\hline $\mathrm{C}$ & 1.916099000 & -1.64 & 0000 \\
\hline & & & \\
\hline $\mathrm{C}$ & 1.384781000 & 0.105097000 & 6000 \\
\hline C & 0.830319000 & & \\
\hline $\mathrm{C}$ & 1.055563000 & 2.345018000 & 57000 \\
\hline $\mathrm{C}$ & 2.702109000 & 0.595 & 4000 \\
\hline $\mathrm{C}$ & 2.086047000 & 2.093817000 & -0.370439000 \\
\hline $\mathrm{C}$ & 2.342914000 & -0.212297000 & 0.910492000 \\
\hline $\mathrm{C}$ & 1.751966000 & 0.328189000 & 2.028890000 \\
\hline $\mathrm{C}$ & 1.195970000 & 1.699509000 & 2.009939000 \\
\hline $\mathrm{C}$ & & & \\
\hline $\mathrm{C}$ & 2.035188000 & -1.568806000 & 0.545085000 \\
\hline
\end{tabular}




\begin{tabular}{|c|c|c|c|}
\hline C & -0.022217000 & 3.146128000 & \\
\hline $\mathrm{C}$ & 0.096599000 & & \\
\hline & 1.218767000 & -2.365340000 & 1.348414000 \\
\hline & 0.988807000 & -2.507763000 & -1.477849000 \\
\hline & -1.283487000 & & -1.569827000 \\
\hline & -1.052798000 & 3.245334000 & 37000 \\
\hline $\mathrm{C}$ & 0.891838000 & -0.475388000 & 2.867937000 \\
\hline & 0.010121000 & & 422000 \\
\hline $\mathrm{C}$ & 0.216495000 & -2.061573000 & 148000 \\
\hline $\mathrm{C}$ & -1.514668000 & 2.215581000 & -2.6 \\
\hline $\mathrm{C}$ & -1.096680000 & 2.470170000 & 2.473468000 \\
\hline 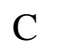 & 0.634364000 & -1.806512000 & 96000 \\
\hline C & -0.737 & 0.02776 & 8000 \\
\hline $\mathrm{C}$ & -0.17 & & \\
\hline $\mathrm{C}$ & 2000 & 0.826 & -0.6 \\
\hline $\mathrm{N}$ & 4.52 & & \\
\hline 0 & $3.31 \varepsilon$ & 3.023 & 05000 \\
\hline $\mathrm{C}$ & $4.79^{\prime}$ & & \\
\hline $\mathrm{H}$ & 4.29 & 0.99 & 3000 \\
\hline $\mathrm{H}$ & 3.48 & & \\
\hline $\mathrm{H}$ & 3.94 & & \\
\hline $\mathrm{H}$ & 4.97 & & 000 \\
\hline $\mathrm{C}$ & 5.26 & -0.2 & 2000 \\
\hline $\mathrm{C}$ & 6.15 & -0.60 & -1.2 \\
\hline $\mathrm{C}$ & 7.1 & -1.5 & 7000 \\
\hline $\mathrm{N}$ & 7.27 & -2.1 & 3000 \\
\hline $\mathrm{C}$ & 6.42 & -1.7 & 000 \\
\hline $\mathrm{C}$ & 5.40 & -0.79 & 000 \\
\hline $\mathrm{H}$ & 6.08 & -0.20 & 9000 \\
\hline $\mathrm{H}$ & 7.83 & $-1.8^{\prime}$ & -1.7 \\
\hline $\mathrm{H}$ & 6.55 & -2.20 & 9000 \\
\hline $\mathrm{H}$ & 5.68 & & 1.6 \\
\hline $\mathrm{H}$ & 4.74 & -0.56 & 5000 \\
\hline $\mathrm{H}$ & 5.352100000 & 2.581240000 & 5000 \\
\hline \multicolumn{4}{|c|}{$\mathrm{ImC}_{60} / \mathrm{b} 31 y p / 6-311 \mathrm{G}(\mathrm{d}, \mathrm{p})$} \\
\hline $\mathrm{H}$ & -1.568506000 & 4.7141 & -0.45 \\
\hline $\mathrm{C}$ & -0.170 & -2.952968000 & 51000 \\
\hline $\mathrm{C}$ & & -3.415657000 & 240000 \\
\hline $\mathrm{C}$ & -0.330 & -3.039 & 82000 \\
\hline $\mathrm{C}$ & & -2.162 & 26000 \\
\hline $\mathrm{C}$ & 1.920 & -0.813 & 139000 \\
\hline $\mathrm{C}$ & 3.242 & -0.267883000 & -3.208884000 \\
\hline $\mathrm{C}$ & & -3.684537000 & -0.531862000 \\
\hline $\mathrm{C}$ & 2.197783000 & -3.591812000 & 0.907783000 \\
\hline $\mathrm{C}$ & & -2.445117000 & -2.331264000 \\
\hline $\mathrm{C}$ & 3.242533000 & -3.190274000 & -1.156637000 \\
\hline $\mathrm{C}$ & & -2.792808000 & -0.104405000 \\
\hline $\mathrm{C}$ & 4.010506000 & -1.273821000 & -2.505223000 \\
\hline
\end{tabular}




\begin{tabular}{|c|c|c|c|}
\hline z & 4.894496000 & -0.897318000 & \\
\hline & 4.967620000 & -1.671325000 & -0.270597000 \\
\hline & 3.512725000 & -3.040116000 & 1.170920000 \\
\hline & 3.696583000 & -2.152071000 & 2.228469000 \\
\hline & 5.165049000 & & .831532000 \\
\hline & 4.540792000 & -0.980837000 & .055318000 \\
\hline & 5.047039000 & 7000 & 15000 \\
\hline & 5.214149000 & & \\
\hline & 2.574086000 & -1.77 & \\
\hline & 2.718565000 & 0000 & 7000 \\
\hline & 3.938 & & \\
\hline & 1.118 & -3.2 & 000 \\
\hline & 1.310 & & \\
\hline & 0.7121060 & -2.6 & 4000 \\
\hline & 4.63 & & \\
\hline & 3.98 & 1.4 & \\
\hline & 3.38 & & \\
\hline & 4.31 & 1.47 & \\
\hline & & & \\
\hline & 3.87 & 2.6 & \\
\hline & 1.64 & 1.79 & \\
\hline & $0.2^{\top}$ & & \\
\hline & -1.49 & -0.8 & 000 \\
\hline 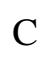 & 0 & & \\
\hline & -0.50 & & 000 \\
\hline$U$ & 0.35 & & \\
\hline & 0.28 & 2.8 & \\
\hline$C$ & -1760 & & \\
\hline . & -0.82 & & \\
\hline 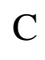 & -1.65 & 0.3 & 000 \\
\hline$C$ & -1.02 & & \\
\hline( & -0.16 & & \\
\hline $\mathrm{C}$ & 0.01 & & \\
\hline & -1.657302000 & & \\
\hline C & 1500 & 3.28 & \\
\hline & .331312000 & & \\
\hline $\mathrm{C}$ & -1.09 & -2.0 & -0 \\
\hline $\mathrm{C}$ & -0.76 & & \\
\hline $\mathrm{C}$ & 2.746 & 3.02 & 7000 \\
\hline $\mathrm{C}$ & 2.417865000 & 2.8497 & 6000 \\
\hline $\mathrm{C}$ & 7000 & & 000 \\
\hline $\mathrm{C}$ & 0.951261000 & & 17000 \\
\hline C & & & \\
\hline $\mathrm{C}$ & 2.819932000 & 2.265154000 & 2.339537000 \\
\hline $\mathrm{C}$ & 2.223396000 & 1.935808000 & -2.797440000 \\
\hline $\mathrm{C}$ & -0.456667000 & -1.785272000 & -2.243800000 \\
\hline C & 1.594406000 & 0.446299000 & 3.459273000 \\
\hline $\mathrm{C}$ & 0.801642000 & 0.008221000 & -3.366580000 \\
\hline $\mathrm{C}$ & -3.201193000 & 2.090013000 & 0.562939000 \\
\hline
\end{tabular}




\begin{tabular}{|c|c|c|c|}
\hline & -3.171002000 & 3.385005000 & \\
\hline $\mathrm{C}$ & & & 0.201999000 \\
\hline & -3.549577000 & 3.458934000 & -1.524285000 \\
\hline & -3.168860000 & 2.332292000 & 1.630363000 \\
\hline & -1.849012000 & 4.271918000 & 1.230104000 \\
\hline & -2.936070000 & 2.850184000 & -2.202552000 \\
\hline & -3.470572000 & 4.500714000 & -1.840771000 \\
\hline & -4.444913000 & & 0.363765000 \\
\hline & -5.351088000 & & 1.425539000 \\
\hline & -6.523626000 & & 50000 \\
\hline C & -6.827641000 & -0.238186000 & 0.120864000 \\
\hline & -5.941717000 & -0.141597000 & 12000 \\
\hline C & 62687000 & 2000 & 2000 \\
\hline & -5.130994000 & & 3000 \\
\hline & -7.190385000 & 0.31 & 4000 \\
\hline & 698000 & -0.61 & 8000 \\
\hline & 627000 & 3.16 & 9000 \\
\hline & 331000 & & \\
\hline & -8.022897000 & -0.99 & -0.0 \\
\hline $\mathrm{C}$ & -9.266880000 & -0.6 & 000 \\
\hline C & 550000 & -1.7 & 9000 \\
\hline & 6000 & -2.6 & 9000 \\
\hline & -8.1 & -2.19 & 000 \\
\hline & 00000 & -1.8 & 2000 \\
\hline & -9. & & 1000 \\
\hline & -7.348523000 & -2.689560000 & 2000 \\
\hline \multicolumn{4}{|c|}{$\mathrm{ImH}^{+} \mathrm{C}_{60} / \mathrm{b} 31 y p / 6-311 \mathrm{G}(\mathrm{d}, \mathrm{p})$} \\
\hline $\mathrm{H}$ & -1.478788000 & 4.771842000 & 761000 \\
\hline & -0.2 & -2.9 & 7000 \\
\hline c & & -3.3 & 6000 \\
\hline & -0. & -3.0 & 000 \\
\hline & & -2.1 & -2.9 \\
\hline c & & -0.7 & -3.4 \\
\hline & & -0.27 & 7000 \\
\hline C & 67470000 & -3.69 & 8000 \\
\hline $\mathrm{C}$ & & -3.612 & 61000 \\
\hline $\mathrm{C}$ & 3.120464000 & -2.45 & 00000 \\
\hline & & -3.213531000 & 17000 \\
\hline C & 3436000 & -2.84096 & 5000 \\
\hline & & -1.300937000 & 27000 \\
\hline $\mathrm{C}$ & 6000 & -0.94 & -1.513 \\
\hline $\mathrm{C}$ & & -1.732265000 & -0.293875000 \\
\hline C & & -3.086207000 & 1.141095000 \\
\hline $\mathrm{C}$ & 3.668229000 & -2.209782000 & 2.204588000 \\
\hline $\mathrm{C}$ & & -0.820899000 & 0.814644000 \\
\hline $\mathrm{C}$ & 4.532620000 & -1.052542000 & 2.038372000 \\
\hline & & & -1.158945000 \\
\hline c & 5.228229000 & 0.525411000 & 0.280820000 \\
\hline
\end{tabular}




\begin{tabular}{|c|c|c|c|}
\hline & 54652000 & -1.821410000 & \\
\hline & & & \\
\hline & 3.952623000 & 0.050157000 & \\
\hline & 1.070247000 & -3.245057000 & 10000 \\
\hline & 1.281336000 & -2.329205000 & 2.797532000 \\
\hline & 0.663003000 & -2.609704000 & -2.35 \\
\hline & 4.675159000 & 1.587071000 & \\
\hline & 4.022089000 & 68000 & 2000 \\
\hline & 3.404167000 & & \\
\hline & 4.335872000 & & \\
\hline & 3.756358000 & 75000 & \\
\hline & 3.9224 & & \\
\hline 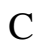 & 35668000 & & \\
\hline 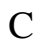 & 0.290187000 & -0.1 & 000 \\
\hline$C$ & 5000 & & 000 \\
\hline C & 000 & & \\
\hline 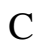 & 0000 & 2000 & 2.50 \\
\hline 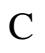 & 8000 & & \\
\hline 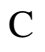 & 5000 & & 000 \\
\hline$C$ & -1.72 & & \\
\hline C & -0.768 & & 3000 \\
\hline$C$ & -1.648 & & \\
\hline$C$ & -1.00 & & -1. \\
\hline$C$ & -0.13 & & -2 \\
\hline$C$ & 0.06 & & \\
\hline & 326000 & & \\
\hline C & 1.5 & & \\
\hline & 000 & & \\
\hline$C$ & 3000 & & 000 \\
\hline & -0.791 & & \\
\hline $\mathrm{C}$ & 47000 & & 000 \\
\hline & 000 & & \\
\hline $\mathrm{C}$ & -0.421 & 000 & 3000 \\
\hline & & & \\
\hline$C$ & 000 & 000 & 1000 \\
\hline $\mathrm{C}$ & 5000 & & \\
\hline$C$ & & & 000 \\
\hline $\mathrm{C}$ & 77000 & +3000 & 0000 \\
\hline & & & \\
\hline $\mathrm{C}$ & 796664000 & 0.045075000 & 6389000 \\
\hline C & -3.147319000 & & 4717000 \\
\hline $\mathrm{N}$ & -3.107583000 & 3.467669000 & -0.082986000 \\
\hline $\mathrm{C}$ & -1.776158000 & 32000 & 76000 \\
\hline $\mathrm{C}$ & -3.471283000 & 3.556877000 & -1.498765000 \\
\hline $\mathrm{H}$ & -3.120189000 & 2.405090000 & 1.653588000 \\
\hline $\mathrm{H}$ & -1.770 & & 1.271081000 \\
\hline $\mathrm{H}$ & -2.874814000 & 2.929422000 & -2.174693000 \\
\hline $\mathrm{H}$ & & 4.595263000 & -1.810553000 \\
\hline $\mathrm{C}$ & -4.396277000 & 1.339786000 & 0.374431000 \\
\hline
\end{tabular}




$\begin{array}{lllc}\mathrm{C} & -5.275643000 & 1.180409000 & 1.453021000 \\ \mathrm{C} & -6.434716000 & 0.419937000 & 1.345269000 \\ \mathrm{C} & -6.721471000 & -0.185861000 & 0.127147000 \\ \mathrm{C} & -5.880636000 & -0.038704000 & -0.971598000 \\ \mathrm{C} & -4.720470000 & 0.716450000 & -0.838558000 \\ \mathrm{H} & -5.044961000 & 1.650146000 & 2.401513000 \\ \mathrm{H} & -7.084804000 & 0.284120000 & 2.201351000 \\ \mathrm{H} & -6.131966000 & -0.488067000 & -1.925321000 \\ \mathrm{H} & -4.525536000 & 3.306222000 & -1.624741000 \\ \mathrm{H} & -4.067871000 & 0.815175000 & -1.693702000 \\ \mathrm{~N} & -7.918201000 & -0.988577000 & 0.002001000 \\ \mathrm{C} & -9.209617000 & -0.583663000 & 0.310255000 \\ \mathrm{C} & -10.036104000 & -1.630178000 & 0.045195000 \\ \mathrm{~N} & -9.233593000 & -2.651544000 & -0.421847000 \\ \mathrm{C} & -7.958270000 & -2.249630000 & -0.437091000 \\ \mathrm{H} & -11.103798000 & -1.729171000 & 0.137236000 \\ \mathrm{H} & -9.419904000 & 0.409691000 & 0.666159000 \\ \mathrm{H} & -7.105782000 & -2.839542000 & -0.728756000 \\ \mathrm{H} & -9.549781000 & -3.571045000 & -0.700693000\end{array}$

\section{$\operatorname{ImC}_{60} \mathrm{H}^{+} / \mathrm{b} 31 \mathrm{yp} / 6-311 \mathrm{G}(\mathrm{d}, \mathrm{p})$}

$\begin{array}{lrrr}\mathrm{H} & -1.558728000 & 4.676384000 & -0.486876000 \\ \mathrm{C} & -0.117760000 & -2.974794000 & 1.116470000 \\ \mathrm{C} & 0.854959000 & -3.433527000 & -1.097998000 \\ \mathrm{C} & -0.272343000 & -3.068689000 & -0.261892000 \\ \mathrm{C} & 1.931843000 & -2.169560000 & -2.916713000 \\ \mathrm{C} & 1.959064000 & -0.821784000 & -3.457983000 \\ \mathrm{C} & 3.273166000 & -0.259064000 & -3.199978000 \\ \mathrm{C} & 2.095802000 & -3.682354000 & -0.517258000 \\ \mathrm{C} & 2.257662000 & -3.582943000 & 0.922526000 \\ \mathrm{C} & 3.221948000 & -2.434359000 & -2.316887000 \\ \mathrm{C} & 3.303172000 & -3.174025000 & -1.139629000 \\ \mathrm{C} & 4.211952000 & -2.761838000 & -0.086350000 \\ \mathrm{C} & 4.051455000 & -1.253134000 & -2.492201000 \\ \mathrm{C} & 4.926553000 & -0.861813000 & -1.481852000 \\ \mathrm{C} & 5.005668000 & -1.630797000 & -0.253714000 \\ \mathrm{C} & 3.564437000 & -3.013254000 & 1.187593000 \\ \mathrm{C} & 3.733967000 & -2.119835000 & 2.242345000 \\ \mathrm{C} & 5.188069000 & -0.701168000 & 0.845644000 \\ \mathrm{C} & 4.563633000 & -0.938528000 & 2.067936000 \\ \mathrm{C} & 5.061048000 & 0.541666000 & -1.142177000 \\ \mathrm{C} & 5.222332000 & 0.640736000 & 0.297615000 \\ \mathrm{C} & 2.604076000 & -1.756599000 & 3.071022000 \\ \mathrm{C} & 2.729428000 & -0.348433000 & 3.404535000 \\ \mathrm{C} & 3.945471000 & 0.153992000 & 2.788753000 \\ \mathrm{C} & 1.172026000 & -3.239886000 & 1.724398000 \\ \mathrm{C} & 1.348770000 & -2.305830000 & 2.821936000 \\ \mathrm{C} & 0.771049000 & -2.660856000 & -2.324328000 \\ \mathrm{C} & 4.634750000 & 1.693334000 & 0.994201000\end{array}$




\begin{tabular}{|c|c|c|c|}
\hline & .981300000 & 444554000 & \\
\hline & 3.404240000 & 089994000 & \\
\hline & 4.317728000 & 1.498973000 & \\
\hline & 3.700485000 & .591071000 & -1.099567000 \\
\hline & 3.855514000 & 685836000 & \\
\hline & 1.628729000 & 4000 & 2.91 \\
\hline & 0.288451000 & -0.111280000 & \\
\hline & -1.470525000 & 3000 & 8000 \\
\hline & 1.376840000 & & \\
\hline & -0.498525000 & & \\
\hline 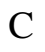 & 0.345621000 & 72195000 & 50000 \\
\hline & 0.272786000 & 20214000 & \\
\hline$C$ & -1.768919000 & 1.3520 & 6000 \\
\hline & -0.8 & & 000 \\
\hline 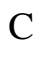 & -1.646934000 & 000 & -0.8 \\
\hline & -1.00 & & \\
\hline & -0.15879 & 1.7 & -2.2 \\
\hline & 0.00 & & -1.1 \\
\hline C & -1.630765000 & -1.007 & -0.2 \\
\hline & 1.4769510 & & \\
\hline$C$ & 1.3 & & -0.9 \\
\hline & -1.0 & & -0 . \\
\hline$C$ & -0.73 & -1.8 & \\
\hline & 2.72 & & \\
\hline$c$ & 2.40 & & -1.7 \\
\hline & -0.37 & -0.52 & -2.7 \\
\hline 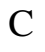 & 0.96 & & -3.0 \\
\hline & 0.168 & -1.4 & 2.8 \\
\hline$C$ & 2.800890000 & 2.2 & 2000 \\
\hline & 2000 & & -2.7 \\
\hline $\mathrm{C}$ & -0.40 & & -2 \\
\hline $\mathrm{C}$ & & & \\
\hline $\mathrm{C}$ & 000 & -0.0 & -3.3 \\
\hline $\mathrm{C}$ & 00 & & \\
\hline IV & -3.182 & & -0.1 \\
\hline $\mathrm{C}$ & -1.82 & & 3000 \\
\hline $\mathrm{C}$ & -3.482276000 & 3.3180 & 38000 \\
\hline $\mathrm{H}$ & -3.182929000 & 99000 & 6000 \\
\hline $\mathrm{H}$ & -1.886036000 & & 10000 \\
\hline $\mathrm{H}$ & -2.745901000 & 0000 & -2.1 \\
\hline $\mathrm{H}$ & -3.434183000 & & -1.9 \\
\hline $\mathrm{C}$ & -4.454587000 & 1000 & 0.365997000 \\
\hline $\mathrm{C}$ & -5.392673000 & & 1.408444000 \\
\hline C & -6.58 & 2000 & 1000 \\
\hline $\mathrm{C}$ & -6.885404000 & -0.258651000 & 0.124598000 \\
\hline $\mathrm{C}$ & -5.959734000 & -0.244623000 & -0.927994000 \\
\hline $\mathrm{C}$ & -4.759437000 & 0.438881000 & -0.802178000 \\
\hline $\mathrm{H}$ & -5.177310000 & 1.656575000 & 2.337244000 \\
\hline & -7.271092000 & 0.409181000 & 2.137579000 \\
\hline
\end{tabular}




\begin{tabular}{|c|c|c|c|}
\hline $\mathrm{H}$ & -6.187380000 & -0.757387000 & \\
\hline $\mathrm{H}$ & -4.480342000 & & \\
\hline & -4.063921000 & 0.409845000 & -1.629775000 \\
\hline & -8.090961000 & -0.967155000 & -0.001383000 \\
\hline & -9.306212000 & -0.684812000 & 0.619397000 \\
\hline & -10.165678000 & -1.660061000 & 0.207913000 \\
\hline & -9.528541000 & -2.551407000 & -0.632817000 \\
\hline & -8.306225000 & -2.11 & 5000 \\
\hline $\mathrm{H}$ & -11.208253000 & -1.773709000 & 0.459233000 \\
\hline & -9.445919000 & 0.185558000 & 1.236454000 \\
\hline $\mathrm{H}$ & -7.514421000 & -2.596163000 & -1.299781000 \\
\hline $\mathrm{H}$ & -3.898199000 & 3.896595000 & 0.283656000 \\
\hline \multicolumn{4}{|c|}{$\mathrm{Py}_{3} \mathrm{C}_{60} / \mathrm{b} 31 y p / 6-311 \mathrm{G}(\mathrm{d}, \mathrm{p})$} \\
\hline $\mathrm{H}$ & -3.580043000 & -1.608987000 & 58000 \\
\hline $\mathrm{C}$ & & & -1.24 \\
\hline 0 & 3.26 & 1000 & 1.02 \\
\hline $\mathrm{C}$ & & $3.4^{7}$ & 0000 \\
\hline C & 971000 & 2000 & 2.95 \\
\hline $\mathrm{C}$ & & 0.56 & 1000 \\
\hline 0 & & -0.7 & 51000 \\
\hline $\mathrm{C}$ & & & 000 \\
\hline C & & 2.2 & -0.8 \\
\hline C & 2000 & 0.97 & 000 \\
\hline $\mathrm{C}$ & & & 000 \\
\hline $\mathrm{C}$ & & 0.46 & 0.28 \\
\hline $\mathrm{C}$ & & -0.4 & 000 \\
\hline C & & -1.3 & 2000 \\
\hline $\mathrm{C}$ & & -0.8 & 7000 \\
\hline $\mathrm{C}$ & & $0.9^{\circ}$ & -1.0 \\
\hline $\mathrm{C}$ & & 00 & 000 \\
\hline $\mathrm{C}$ & & -1.8 & -0.5 \\
\hline $\mathrm{C}$ & 000 & -1.3 & 2000 \\
\hline $\mathrm{C}$ & & -2.5 & 3000 \\
\hline $\mathrm{C}$ & & -2.85 & 3000 \\
\hline $\mathrm{C}$ & 294000 & 0.44 & 3000 \\
\hline $\mathrm{C}$ & & -0.760690000 & -3.23 \\
\hline $\mathrm{C}$ & 3.639685000 & -1.85 & 44000 \\
\hline $\mathrm{C}$ & & 2.58 & -1.757 \\
\hline $\mathrm{C}$ & 3.116609000 & 1.66 & 3000 \\
\hline $\mathrm{C}$ & & 2.65 & 2.264732000 \\
\hline $\mathrm{C}$ & 3.157034000 & 868000 & -0.626315000 \\
\hline $\mathrm{C}$ & & -2.859250000 & -1.951676000 \\
\hline $\mathrm{C}$ & & -1.86 & 3.124835000 \\
\hline $\mathrm{C}$ & 2.915562000 & -2.823626000 & 2.155892000 \\
\hline $\mathrm{C}$ & 1.784807000 & -3.354393000 & 1.417541000 \\
\hline $\mathrm{C}$ & 1.902438000 & -3.618864000 & 0.057144000 \\
\hline $\mathrm{C}$ & 0.807476000 & -1.735581000 & -2.756757000 \\
\hline $\mathrm{C}$ & 0.943398000 & 0.566941000 & -3.198035000 \\
\hline
\end{tabular}




\begin{tabular}{|c|c|c|c|}
\hline & -0.023299000 & 409049000 & \\
\hline $\mathrm{C}$ & & & \\
\hline & 0.311629000 & & \\
\hline & .395692000 & & \\
\hline & 0.967421000 & & \\
\hline & 1.687744000 & & 9000 \\
\hline & -1.779382000 & -0.70 & \\
\hline & & & 3000 \\
\hline C & -0.696223000 & & \\
\hline & 3000 & & \\
\hline C & -1.156572000 & & 50000 \\
\hline & & & 18000 \\
\hline & 000 & -2.6 & 6000 \\
\hline $\mathrm{C}$ & & & \\
\hline C & 000 & 3.2 & \\
\hline $\mathrm{C}$ & & & \\
\hline & & -3.2 & \\
\hline $\mathrm{C}$ & & -2.7 & \\
\hline 0 & & & 2000 \\
\hline $\mathrm{C}$ & & -0.5 & \\
\hline C & & & -2.9 \\
\hline C & & -2.8 & \\
\hline $\mathrm{C}$ & & -1.8 & \\
\hline C & & & \\
\hline C & & -0.6 & \\
\hline & & & \\
\hline $\mathrm{C}$ & 00 & & -0.7 \\
\hline $\mathrm{N}$ & -4.0 & & \\
\hline $\mathrm{C}$ & 000 & -1.0 & \\
\hline $\mathrm{C}$ & & & \\
\hline $\mathrm{H}$ & & & 000 \\
\hline $\mathrm{H}$ & & & \\
\hline $\mathrm{C}$ & -3.6 & & -0 . \\
\hline $\mathrm{C}$ & & & \\
\hline $\mathrm{C}$ & & & \\
\hline $\mathrm{N}$ & 0000 & & -0.7 \\
\hline $\mathrm{C}$ & -3.916934000 & & 57000 \\
\hline $\mathrm{C}$ & 45000 & 5000 & 2000 \\
\hline $\mathrm{H}$ & & & \\
\hline $\mathrm{H}$ & -5.14 & & 29000 \\
\hline $\mathrm{H}$ & & & 640000 \\
\hline $\mathrm{H}$ & 1000 & 1.641412000 & 1.324178000 \\
\hline $\mathrm{H}$ & & 3.203153000 & 1.297177000 \\
\hline $\mathrm{C}$ & & & 1.833148000 \\
\hline $\mathrm{C}$ & -5.455030000 & -1.139763000 & 2.916163000 \\
\hline $\mathrm{N}$ & & & 1.167771000 \\
\hline $\mathrm{C}$ & & -1.932291000 & 3.318670000 \\
\hline $\mathrm{C}$ & & -0.933053000 & 1.559968000 \\
\hline $\mathrm{C}$ & -7.727402000 & -1.831463000 & 2.625206000 \\
\hline
\end{tabular}




$\begin{array}{llll}\mathrm{H} & -4.504655000 & -1.188436000 & 3.435802000 \\ \mathrm{H} & -6.426023000 & -2.611570000 & 4.157719000 \\ \mathrm{H} & -8.721192000 & -0.818321000 & 0.999028000 \\ \mathrm{H} & -8.591515000 & -2.424354000 & 2.900294000 \\ \mathrm{C} & -3.859113000 & -1.805553000 & -1.308273000 \\ \mathrm{~N} & -3.776797000 & -1.237600000 & -2.517814000 \\ \mathrm{C} & -4.393700000 & -3.082830000 & -1.124837000 \\ \mathrm{C} & -4.232874000 & -1.915677000 & -3.571553000 \\ \mathrm{C} & -4.858814000 & -3.787560000 & -2.230694000 \\ \mathrm{C} & -4.780524000 & -3.193490000 & -3.484842000 \\ \mathrm{H} & -4.451494000 & -3.512154000 & -0.131626000 \\ \mathrm{H} & -4.150278000 & -1.415745000 & -4.532549000 \\ \mathrm{H} & -5.277321000 & -4.780720000 & -2.112575000 \\ \mathrm{H} & -5.130408000 & -3.701704000 & -4.375086000\end{array}$

$\mathrm{Py}_{3} \mathrm{H}^{+} \mathrm{C}_{60} / \mathrm{b} 31 \mathrm{yp} / 6-311 \mathrm{G}(\mathrm{d}, \mathrm{p})$

$\begin{array}{lrrr}\mathrm{H} & -3.420273000 & -1.917289000 & 0.784313000 \\ \mathrm{C} & 2.191415000 & 3.218362000 & -1.365295000 \\ \mathrm{C} & 3.135898000 & 3.295241000 & 0.905931000 \\ \mathrm{C} & 2.047200000 & 3.538833000 & -0.020539000 \\ \mathrm{C} & 3.198672000 & 1.860001000 & 2.905059000 \\ \mathrm{C} & 2.420194000 & 0.804526000 & 3.529520000 \\ \mathrm{C} & 3.185761000 & -0.426839000 & 3.450355000 \\ \mathrm{C} & 4.323733000 & 2.731993000 & 0.445135000 \\ \mathrm{C} & 4.473945000 & 2.397554000 & -0.960259000 \\ \mathrm{C} & 4.436975000 & 1.279036000 & 2.430090000 \\ \mathrm{C} & 4.987979000 & 1.704157000 & 1.223440000 \\ \mathrm{C} & 5.549519000 & 0.734890000 & 0.301058000 \\ \mathrm{C} & 4.428711000 & -0.135231000 & 2.766874000 \\ \mathrm{C} & 4.970775000 & -1.063702000 & 1.881401000 \\ \mathrm{C} & 5.540000000 & -0.619301000 & 0.622842000 \\ \mathrm{C} & 5.230170000 & 1.162977000 & -1.048365000 \\ \mathrm{C} & 4.909317000 & 0.218033000 & -2.020336000 \\ \mathrm{C} & 5.212608000 & -1.605604000 & -0.390169000 \\ \mathrm{C} & 4.900230000 & -1.196347000 & -1.684135000 \\ \mathrm{C} & 4.293954000 & -2.324408000 & 1.647012000 \\ \mathrm{C} & 4.443322000 & -2.659642000 & 0.241920000 \\ \mathrm{C} & 3.819357000 & 0.466408000 & -2.940123000 \\ \mathrm{C} & 3.131212000 & -0.792471000 & -3.168861000 \\ \mathrm{C} & 3.806391000 & -1.821373000 & -2.397940000 \\ \mathrm{C} & 3.429556000 & 2.639216000 & -1.849534000 \\ \mathrm{C} & 3.094649000 & 1.652719000 & -2.861502000 \\ \mathrm{C} & 2.561029000 & 2.850694000 & 2.162749000 \\ \mathrm{C} & 3.394299000 & -3.265208000 & -0.445573000 \\ \mathrm{C} & 3.067651000 & -2.836117000 & -1.794133000 \\ \mathrm{C} & 2.534915000 & -1.638184000 & 3.228549000 \\ \mathrm{C} & 3.101921000 & -2.608236000 & 2.308081000 \\ \mathrm{C} & 2.008421000 & -3.233400000 & 1.588836000 \\ \mathrm{C} & 2.150870000 & -3.554222000 & 0.243080000\end{array}$




\begin{tabular}{|c|c|c|c|}
\hline C & & 196000 & \\
\hline C & & & \\
\hline & -0.090524000 & & \\
\hline & .808063000 & & 68000 \\
\hline & & & \\
\hline & .267128000 & -1.300415000 & -2.1 \\
\hline$C$ & -0.815886000 & & \\
\hline & 2876000 & & 1000 \\
\hline$C$ & -1.684642000 & & \\
\hline & & & \\
\hline C & -0.72 & & \\
\hline$C$ & 9656000 & & \\
\hline 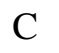 & & & \\
\hline 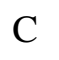 & -0.2 & & \\
\hline$C$ & -0.1 & -2.7 & -0.2 \\
\hline $\mathrm{C}$ & -0.2 & & \\
\hline$C$ & & 3.2 & \\
\hline C & & & -2.0 \\
\hline 0 & & -3.3 & -0.6 \\
\hline $\mathrm{C}$ & & -2.6 & \\
\hline$c$ & & & \\
\hline $\mathrm{C}$ & & -0.4 & \\
\hline $\mathrm{C}$ & & & $-3 .($ \\
\hline C & & -2.8 & -1 . \\
\hline C & & -1.6 & \\
\hline 0 & & & \\
\hline $\mathrm{C}$ & & -0.8 & -3.2 \\
\hline $\mathrm{C}$ & & & \\
\hline $\mathrm{C}$ & & & -0.7 \\
\hline $\mathrm{N}$ & & -0.0 & \\
\hline $\mathrm{C}$ & & & \\
\hline $\mathrm{C}$ & -4.2 & & \\
\hline $\mathrm{H}$ & -3.3 & & -1.7 \\
\hline $\mathrm{H}$ & & & \\
\hline $\mathrm{C}$ & & & -0.8 \\
\hline $\mathrm{C}$ & -5.2 & & -0.5 \\
\hline $\mathrm{C}$ & -5.92 & & -0.7 \\
\hline $\mathrm{N}$ & -5.2 & & -1.2 \\
\hline $\mathrm{C}$ & & & \\
\hline $\mathrm{C}$ & -3.2 & 5000 & 9000 \\
\hline $\mathrm{H}$ & & & -0.2 \\
\hline $\mathrm{H}$ & 50000 & 3.734 & 42000 \\
\hline $\mathrm{H}$ & -3.447687000 & 5.489393000 & 39000 \\
\hline $\mathrm{H}$ & & & 502000 \\
\hline $\mathrm{H}$ & -2.188009000 & 3.384438000 & -1.580383000 \\
\hline $\mathrm{C}$ & & -0.253773000 & 1.975735000 \\
\hline $\mathrm{C}$ & -5.622713000 & -1.175531000 & 3.019919000 \\
\hline $\mathrm{N}$ & & & 1.344506000 \\
\hline $\mathrm{C}$ & -6.864533000 & -1.665143000 & 3.419964000 \\
\hline
\end{tabular}




\begin{tabular}{|c|c|c|c|}
\hline & -7.846985000 & -0.289213000 & \\
\hline & -8.002561000 & -1.216273000 & \\
\hline & -4.714208000 & & \\
\hline & -6.938221000 & -2.382813000 & .228735000 \\
\hline & -8.714605000 & 0.088080000 & .199531000 \\
\hline & -8.989280000 & -1.568604000 & 4000 \\
\hline & -3.688685000 & -2.064965000 & -1.30 \\
\hline & -3.7330 & -1.41 & 3000 \\
\hline & -4.034174000 & -3.411821000 & -1.193422000 \\
\hline & -4.124972000 & -2.087404000 & \\
\hline & -4.434336000 & -4.106035000 & -2.33 \\
\hline & -4.482421000 & -3.432777000 & \\
\hline & -3.99 & -3.90 & -0.2 \\
\hline & -4.14 & -1.52 & \\
\hline & -4.705 & -5.153338000 & -2.2 \\
\hline & -4.78 & -3.93 & \\
\hline & -5.725492000 & 5.5352 & \\
\hline \multicolumn{4}{|c|}{$\mathrm{Py}_{3} \mathrm{C}_{60} \mathrm{H}^{+} / \mathrm{b} 31 y p / 6-311 \mathrm{G}(\mathrm{d}, \mathrm{p})$} \\
\hline 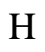 & -3.480808000 & -1.656838000 & \\
\hline & & & \\
\hline & 3.23 & 3.26 & \\
\hline & 2.13 & & -0.2 \\
\hline & 3.2 & & \\
\hline & $2.4 \mathrm{C}$ & & \\
\hline & 3.22 & -0.3 & \\
\hline & 4.39 & 2.64 & \\
\hline & 4.5 & 2.22 & -1.1 \\
\hline & 4.50 & 1.29 & \\
\hline & 5.04 & 1.6 & \\
\hline & & 0.60 & \\
\hline & 4.45 & -0.09 & \\
\hline & & -1.0 & \\
\hline & & -0.73 & 000 \\
\hline & 5.22 & 0.96 & -1.1 \\
\hline & & -0.020396000 & \\
\hline & 16000 & -1.76 & -0.38 \\
\hline & & -1.41 & -1.68 \\
\hline & 4.236 & 445000 & 1.708744000 \\
\hline & & -2.755589000 & \\
\hline & 3.755 & 0.212267000 & -3.0 \\
\hline & & -1.03 & -3.16 \\
\hline 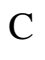 & 3.682614000 & -2.040922000 & -2.347790000 \\
\hline & 3.452591000 & 2.452025000 & -2.039061000 \\
\hline & 3.067 & 1.423294000 & -2.988722000 \\
\hline & 2.671433000 & 2.906316000 & 1.968881000 \\
\hline & & -3.364885000 & -0.313069000 \\
\hline $\mathrm{C}$ & 2.926886000 & -2.999202000 & -1.676686000 \\
\hline & & -1.515385000 & 3.282994000 \\
\hline & 3.050121000 & -2.550854000 & 2.406894000 \\
\hline
\end{tabular}




\begin{tabular}{|c|c|c|c|}
\hline & 000 & 78690000 & \\
\hline & 2.029659000 & -3.575451000 & \\
\hline & .847526000 & -2.011091000 & \\
\hline & .918538000 & 0.238648000 & \\
\hline & .070192000 & 2.240625000 & -1.525867000 \\
\hline & .809775000 & 1.025644000 & -1.695872000 \\
\hline & .319635000 & 0.036804000 & \\
\hline & 65000 & 0000 & 1000 \\
\hline & -0.908131000 & & \\
\hline & 30000 & & \\
\hline & -1.733425000 & 2000 & 5000 \\
\hline & -1.005499000 & & \\
\hline & -0.664 & & 000 \\
\hline & -0.708 & & \\
\hline & -1.076918000 & -1.0 & 000 \\
\hline & -0.183140000 & & \\
\hline & -0.2287 & -2.7 & \\
\hline & -0.33 & & \\
\hline & 0.8966 & 3.27 & \\
\hline & 1.115 & & \\
\hline & 0.930 & -3.3 & \\
\hline & 0.714 & -2.5 & \\
\hline$C$ & 0.46 & & \\
\hline & 0.390 & -0.2 & \\
\hline 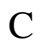 & 1.62 & & \\
\hline & 1.48 & -2.9 & -1 . \\
\hline$C$ & 1.08 & -1.5 & \\
\hline & 1.22760 & & \\
\hline$C$ & 1.637454000 & -1.0 & \\
\hline & 1.111 & & \\
\hline$C$ & -3.170 & & 000 \\
\hline$N$ & -4.011 & & \\
\hline$C$ & -3.27510 & -1.1 & 000 \\
\hline 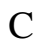 & -4.37 & & \\
\hline H & -3.331004000 & & 000 \\
\hline $\mathrm{H}$ & -3.52406 & & 1000 \\
\hline C & -3.683624000 & & -0.943618000 \\
\hline $\mathrm{C}$ & -4.403587000 & 2.825 & -2.0916 \\
\hline $\mathrm{C}$ & -4.902518000 & & -2.224141000 \\
\hline $\mathrm{N}$ & -4.732206000 & & -1.303970000 \\
\hline $\mathrm{C}$ & -4.040829000 & & -0.210071000 \\
\hline $\mathrm{C}$ & -3.499234000 & 3.480 & 0.018688000 \\
\hline $\mathrm{H}$ & -4.573193000 & & -2.878516000 \\
\hline $\mathrm{H}$ & -5.461 & & -3.110762000 \\
\hline $\mathrm{H}$ & -3.905812000 & & 0.523741000 \\
\hline $\mathrm{H}$ & -4.591283000 & & 1.363125000 \\
\hline $\mathrm{H}$ & -2.934448000 & 3.305836000 & 0.924231000 \\
\hline C & -5.612844000 & -0.102707000 & 1.910063000 \\
\hline & -5.877656000 & -0.314101000 & 3.260242000 \\
\hline
\end{tabular}




\begin{tabular}{|c|c|c|c|}
\hline $\mathrm{N}$ & -6.445876000 & -0.473078000 & \\
\hline $\mathrm{C}$ & -7.080907000 & -0.925806000 & \\
\hline$C$ & -7.595555000 & -1.066291000 & 1.270501000 \\
\hline & -7.958326000 & -1.307052000 & 2.592686000 \\
\hline & -5.169885000 & -0.006998000 & 4.021273000 \\
\hline & -7.326143000 & -1.104430000 & 4.643569000 \\
\hline $\mathrm{H}$ & -8.244371000 & -1.355730000 & 0.450806000 \\
\hline & -8.902129000 & & 116000 \\
\hline $\mathrm{C}$ & -3.814223000 & & -1.056080000 \\
\hline $\mathrm{N}$ & -4.499875000 & & -2.02 \\
\hline $\mathrm{C}$ & -3.615274000 & -3.432137000 & -1.022189000 \\
\hline 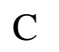 & -5.001261000 & -2.187711000 & -3.01 \\
\hline $\mathrm{C}$ & -4.13 & -4.1 & 9000 \\
\hline $\mathrm{C}$ & -4.83 & & -3.0 \\
\hline $\mathrm{H}$ & 902000 & -3.8 & -0.2 \\
\hline $\mathrm{H}$ & -5.55 & & \\
\hline $\mathrm{H}$ & -3.997024000 & -5.2 & -2.0 \\
\hline $\mathrm{H}$ & -5.25 & & -3.9 \\
\hline $\mathrm{H}$ & -4.919 & 2000 & -0.4 \\
\hline \multicolumn{4}{|c|}{$\mathrm{Py}_{3} \mathrm{H}^{+}{ }_{2-\mathrm{Py}} \mathrm{C}_{60} / \mathrm{b} 31 \mathrm{yp} / 6-311 \mathrm{G}(\mathrm{d}, \mathrm{p})$} \\
\hline $\mathrm{H}$ & -2.957961000 & -1.619177000 & 2.35 \\
\hline 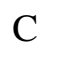 & 1.5 & & -2.3 \\
\hline $\mathrm{C}$ & 2.95 & & -0.4 \\
\hline $\mathrm{C}$ & & & -1.1 \\
\hline C & 3.54 & & 1.8 \\
\hline $\mathrm{C}$ & 3.01 & & 2.8 \\
\hline C & 3.86 & & 2.97 \\
\hline $\mathrm{C}$ & 000 & & -0.9 \\
\hline $\mathrm{C}$ & 3.97 & & -2.2 \\
\hline C & & & \\
\hline $\mathrm{C}$ & 4.9 & & -0.0 \\
\hline $\mathrm{C}$ & .000 & & -0.8 \\
\hline $\mathrm{C}$ & & & 00 \\
\hline $\mathrm{C}$ & & & 1.28 \\
\hline $\mathrm{C}$ & 18000 & -0.2 & -0.13 \\
\hline $\mathrm{C}$ & 4.818941000 & & -2.12 \\
\hline $\mathrm{C}$ & 4.408499000 & -0.2 & 89000 \\
\hline $\mathrm{C}$ & & & -0.75 \\
\hline $\mathrm{C}$ & 45000 & -1.484448000 & -2.00 \\
\hline $\mathrm{C}$ & 52000 & & 5000 \\
\hline $\mathrm{C}$ & 5000 & -2.355070000 & 275000 \\
\hline $\mathrm{C}$ & 3.141319000 & -0.291612000 & -3.405523000 \\
\hline $\mathrm{C}$ & & & 865000 \\
\hline $\mathrm{C}$ & 3.458046000 & -2.327253000 & -2.273218000 \\
\hline $\mathrm{C}$ & 2.761590000 & 2.085042000 & -2.883022000 \\
\hline $\mathrm{C}$ & 2.333495000 & 0.839394000 & -3.494490000 \\
\hline $\mathrm{C}$ & & & 1.010458000 \\
\hline $\mathrm{C}$ & 3.579464000 & -3.171024000 & 0.037254000 \\
\hline
\end{tabular}




\begin{tabular}{|c|c|c|c|}
\hline & 55125000 & 156641000 & \\
\hline C & & & 3.232153000 \\
\hline & 769891000 & & \\
\hline & 622220000 & -2.613029000 & 2.235610000 \\
\hline & & & 1.029595000 \\
\hline & 646885000 & -2.556136000 & -1.901801000 \\
\hline & 329615000 & -0.523584000 & -3.0 \\
\hline & 557132000 & 129000 & 5000 \\
\hline & & & -1.3 \\
\hline & .724701000 & 32000 & 5000 \\
\hline C & -0.524331000 & & -1.337 \\
\hline & 8000 & & \\
\hline c & 000 & & 000 \\
\hline & 000 & & \\
\hline C & 000 & 1.6 & 0.6 \\
\hline & 000 & & \\
\hline & -0.2 & & 000 \\
\hline $\mathrm{C}$ & -0.6 & & \\
\hline 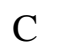 & -0.45 & & -0.3 \\
\hline $\mathrm{C}$ & & -2.7 & \\
\hline & & & 2.1 \\
\hline C & & & -0.1 \\
\hline C & & & -2.5 \\
\hline C & 000 & -3.3 & 0.3 \\
\hline C & & & \\
\hline C & & & $2.1^{\prime}$ \\
\hline $\mathrm{C}$ & & & \\
\hline $\mathrm{C}$ & & & -3.3 \\
\hline $\mathrm{C}$ & 000 & -3.2 & -1.0 \\
\hline C & & -0.7 & \\
\hline C & & & \\
\hline $\mathrm{C}$ & & -1. & -2.9 \\
\hline $\mathrm{C}$ & & & 3.0 \\
\hline $\mathrm{C}$ & & & -0.0 \\
\hline $\mathrm{N}$ & & & \\
\hline $\mathrm{C}$ & 000 & & 1.28 \\
\hline $\mathrm{C}$ & -5.150862000 & & 1.6288 \\
\hline $\mathrm{H}$ & 9000 & 000 & 778000 \\
\hline $\mathrm{H}$ & & & 2.209275000 \\
\hline $\mathrm{C}$ & -3.816 & 8000 & 0000 \\
\hline $\mathrm{C}$ & -4.24 & & -1.13 \\
\hline $\mathrm{C}$ & -4.67 & 4.001653000 & -1.069826000 \\
\hline $\mathrm{N}$ & -4.726 & & 0.060373000 \\
\hline $\mathrm{C}$ & & & 16000 \\
\hline $\mathrm{C}$ & -3.843417000 & 2.791147000 & 1.216309000 \\
\hline $\mathrm{H}$ & & & -2.084531000 \\
\hline $\mathrm{H}$ & -5.009133000 & & -1.969594000 \\
\hline $\mathrm{H}$ & -4.338016000 & 4.699942000 & 2.083260000 \\
\hline $\mathrm{H}$ & -5.240066000 & 0.743406000 & 2.327128000 \\
\hline
\end{tabular}




\begin{tabular}{|c|c|c|c|}
\hline $\mathrm{H}$ & -3.496119000 & 2.366149000 & \\
\hline $\mathrm{C}$ & & & 0.603274000 \\
\hline & -7.320708000 & & 0.773038000 \\
\hline & -6.193130000 & -0.640072000 & -0.532387000 \\
\hline & -8.289968000 & & -0.216539000 \\
\hline & -7.110747000 & -0.544798000 & -1.513385000 \\
\hline $\mathrm{C}$ & -8.183785000 & 0.312572000 & \\
\hline & -7.371224000 & & 1.670651000 \\
\hline $\mathrm{H}$ & -9.120034000 & 1.757065000 & -0.087741000 \\
\hline $\mathrm{H}$ & -6.951898000 & -1.166696000 & \\
\hline $\mathrm{H}$ & -8.915285000 & 0.387353000 & \\
\hline & -3.500980000 & -2.685610000 & 0.614136000 \\
\hline 1 & -4.34 & -2.61 & 5000 \\
\hline 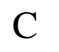 & -3.04 & -3.5 & \\
\hline C & -4.714513000 & -3.74 & -1.0 \\
\hline $\mathrm{C}$ & -3.42 & -5.08 & \\
\hline $\mathrm{C}$ & -4.270 & -5.00 & -0.6 \\
\hline $\mathrm{H}$ & -2.38 & & \\
\hline $\mathrm{H}$ & -5.39 & -3.6 & 9000 \\
\hline $\mathrm{H}$ & -3.0 & & \\
\hline $\mathrm{H}$ & -4.58 & -5.8 & 2000 \\
\hline $\mathrm{H}$ & -5.38 & -1.35 & 3000 \\
\hline \multicolumn{4}{|c|}{$\mathrm{Py}_{3} \mathrm{H}^{+}{ }_{3-\mathrm{Py}} \mathrm{C}_{60} / \mathrm{b} 31 \mathrm{lyp} / 6-311 \mathrm{G}(\mathrm{d}, \mathrm{p})$} \\
\hline $\mathrm{H}$ & -3.582784000 & -1.552311000 & \\
\hline $\mathrm{C}$ & & & \\
\hline $\mathrm{C}$ & $3.2^{\prime}$ & & \\
\hline $\mathrm{C}$ & 2.19 & & -0.0 \\
\hline $\mathrm{C}$ & 3.27 & & 0000 \\
\hline $\mathrm{C}$ & 2.44 & & \\
\hline C & 3.14 & -0.5 & 3000 \\
\hline $\mathrm{C}$ & 4.43 & & \\
\hline $\mathrm{C}$ & 4.55 & 2.22 & -0.9 \\
\hline $\mathrm{C}$ & & & \\
\hline $\mathrm{C}$ & & & \\
\hline $\mathrm{C}$ & 000 & 00 & 5000 \\
\hline $\mathrm{C}$ & & -0.30 & 2.73 \\
\hline $\mathrm{C}$ & 4.890 & -1.25 & 27000 \\
\hline $\mathrm{C}$ & & -0.84 & \\
\hline $\mathrm{C}$ & 5.251 & 0.95 & 19000 \\
\hline $\mathrm{C}$ & & & 169000 \\
\hline $\mathrm{C}$ & 5.0952 & -1.809 & -0.417432000 \\
\hline $\mathrm{C}$ & & -1.385 & -1.710957000 \\
\hline $\mathrm{C}$ & & & 1.622899000 \\
\hline $\mathrm{C}$ & 4.275869000 & -2.823474000 & 0.217534000 \\
\hline $\mathrm{C}$ & & 0.329753000 & -2.966673000 \\
\hline $\mathrm{C}$ & 3.050558000 & -0.891460000 & -3.190958000 \\
\hline $\mathrm{C}$ & & -1.953467000 & -2.418850000 \\
\hline $\mathrm{C}$ & 3.525618000 & 2.519740000 & -1.874093000 \\
\hline
\end{tabular}




\begin{tabular}{|c|c|c|c|}
\hline & 7797000 & & \\
\hline & & & \\
\hline & 3.196441000 & -3.376997000 & \\
\hline & 2.888849000 & -2.931972000 & 3901000 \\
\hline & 2.431904000 & -1.704977000 & \\
\hline & .945973000 & -2.702006000 & 44000 \\
\hline & 1.819545000 & -3.269091000 & \\
\hline & 1.942777000 & -3.601732000 & 2000 \\
\hline & 0.845796000 & -1.851793000 & \\
\hline & 15000 & 0.42 & \\
\hline & -0.008642000 & 2.3533 & \\
\hline & 0.768739000 & 1.1622 & \\
\hline$\zeta$ & 11000 & 0.214886000 & \\
\hline & 1000 & -1.2173 & \\
\hline( & -0.932 & -1.6 & -1 . \\
\hline 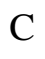 & -1.6 & 0.8 & \\
\hline$\zeta$ & 4000 & -0.71 & 0000 \\
\hline & -0.9 & & \\
\hline & -0.68 & 1.28 & \\
\hline & -0.7 & -0.1 & \\
\hline 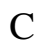 & -1.13 & -1.07 & 1.2 \\
\hline & -0.13 & & \\
\hline 4 & -0.2 & -2.6 & \\
\hline & -0.4 & -2.3 & \\
\hline 4 & 0.9 & 3.2 & \\
\hline & & 2.58 & \\
\hline$C$ & & -3.2 & -0 . \\
\hline & & -2.6 & \\
\hline 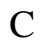 & 00 & 1.8 & 000 \\
\hline & 9000 & -0.4 & \\
\hline$C$ & 000 & 1.60 & -3.0 \\
\hline & & -2.88 & -1. \\
\hline $\mathrm{C}$ & 00 & -1.6 & 000 \\
\hline & & & \\
\hline$C$ & 000 & -0.84 & -3.3 \\
\hline 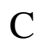 & 4000 & 0.78 & \\
\hline$C$ & & & \\
\hline $\mathrm{N}$ & -4.02 & 0.27 & -0.0 \\
\hline & & -1.00 & \\
\hline $\mathrm{C}$ & -4.5001570 & 0.698 & 8000 \\
\hline $\mathrm{H}$ & -3.286193000 & & 8356000 \\
\hline $\mathrm{H}$ & -3.708604000 & 7000 & \\
\hline $\mathrm{C}$ & $-3.60 s$ & & -0.8 \\
\hline $\mathrm{C}$ & -4.190640000 & 3.122801000 & -2.044879000 \\
\hline $\mathrm{C}$ & -4.593766000 & 4.454419000 & -2.115649000 \\
\hline $\mathrm{N}$ & & & -1.108471000 \\
\hline $\mathrm{C}$ & -3.898117000 & 4.858826000 & 0.012924000 \\
\hline $\mathrm{C}$ & & & 0.182159000 \\
\hline & -4.323838000 & 2.476678000 & -2.905689000 \\
\hline
\end{tabular}




\begin{tabular}{|c|c|c|c|}
\hline $\mathrm{H}$ & -5.044056000 & 4.842613000 & \\
\hline $\mathrm{H}$ & & & 0.821630000 \\
\hline & -4.819682000 & 1.733776000 & 1.240040000 \\
\hline & -3.005201000 & 3.260220000 & .120323000 \\
\hline & -5.676289000 & -0.134273000 & 1.795477000 \\
\hline & -6.949699000 & 0.422815000 & 1.921312000 \\
\hline & -5.421107000 & -1.412801000 & 2.114017000 \\
\hline & -7.988443000 & & \\
\hline & -6.425229000 & -2.166246000 & \\
\hline & -7.723062000 & -1.691194000 & 67000 \\
\hline & -7.121385000 & 1.458994000 & \\
\hline & -8.984079000 & & \\
\hline & -6.176 & 5000 & 5000 \\
\hline & -8.49 & -2.3 & \\
\hline C & -3.90 & 0000 & -1. \\
\hline & & & \\
\hline( & -5.000869000 & 2000 & \\
\hline $\mathrm{C}$ & -3.849303000 & & \\
\hline C & 06098000 & -2.3 & \\
\hline $\mathrm{C}$ & -4.9 & & \\
\hline 1 & & -0.5 & \\
\hline & -3.3 & & 000 \\
\hline F & & & \\
\hline & -5.3 & & 000 \\
\hline $\mathrm{H}$ & -2.5 & 3000 & \\
\hline \multicolumn{4}{|c|}{$\mathrm{Py}_{2} \mathrm{C}_{70} / \mathrm{b} 31 y p / 6-311 \mathrm{G}(\mathrm{d}, \mathrm{p})$} \\
\hline $\mathrm{C}$ & & -0.6546 & 6000 \\
\hline $\mathrm{C}$ & & -3.3 & \\
\hline & & -1.6 & \\
\hline C & & & 5000 \\
\hline $\mathrm{C}$ & & & \\
\hline C & 000 & 00 & 000 \\
\hline $\mathrm{C}$ & & & \\
\hline $\mathrm{C}$ & & & -1.0 \\
\hline $\mathrm{C}$ & 050000 & -0.34 & -3.5 \\
\hline $\mathrm{C}$ & -0.098389000 & & -1.1 \\
\hline $\mathrm{C}$ & 299000 & -1.403644000 & 84000 \\
\hline $\mathrm{C}$ & & & \\
\hline $\mathrm{C}$ & -1.831457000 & 22357000 & 5000 \\
\hline $\mathrm{C}$ & & & \\
\hline $\mathrm{C}$ & 17000 & -2.554115000 & 925000 \\
\hline $\mathrm{C}$ & & & 343000 \\
\hline $\mathrm{C}$ & & 152000 & 6610000 \\
\hline $\mathrm{C}$ & & 1.794987000 & -1.506228000 \\
\hline $\mathrm{C}$ & & -0.610857000 & 0.550074000 \\
\hline $\mathrm{C}$ & & 2.528494000 & -0.267315000 \\
\hline $\mathrm{C}$ & & -0.825028000 & 2.635215000 \\
\hline $\mathrm{C}$ & 4.261117000 & 1.684505000 & 2.034757000 \\
\hline
\end{tabular}




\begin{tabular}{|c|c|c|c|}
\hline & -0.706957000 & -3.264741000 & \\
\hline $\mathrm{C}$ & & & \\
\hline & -1.062309000 & & -1.256337000 \\
\hline & 4.825457000 & -0.6300 & 962000 \\
\hline & -2.206434000 & -0.620 & 22000 \\
\hline & 3.688434000 & 7000 & 1000 \\
\hline & -2.836057000 & & \\
\hline & 3.341812 & 3.322 & 1000 \\
\hline & -0.458433000 & & \\
\hline & 4.013541000 & & \\
\hline C & 0.287223000 & -3.834 & \\
\hline & 4.753892000 & -1.930 & \\
\hline C & -0.64 & -2.4 & 000 \\
\hline & & & \\
\hline C & -1.960107000 & 0.82 & -1.8 \\
\hline $\mathrm{C}$ & & & \\
\hline & -1.8 & 1.4 & 5000 \\
\hline $\mathrm{C}$ & & & \\
\hline 0 & -1.3 & 0.62 & 6000 \\
\hline $\mathrm{C}$ & & 2.50 & \\
\hline$c$ & -0.0 & -2.6 & \\
\hline & & & \\
\hline C & & -3.7 & \\
\hline $\mathrm{C}$ & $4.5^{\circ}$ & -1.8 & \\
\hline C & -1.1 & & \\
\hline & & & -3.2 \\
\hline $\mathrm{C}$ & -2.1 & 1.5 & -0. \\
\hline $\mathrm{C}$ & & 3.41 & -1.1 \\
\hline $\mathrm{C}$ & 000 & -0.43 & 1000 \\
\hline$C$ & & & \\
\hline $\mathrm{C}$ & & & 8000 \\
\hline $\mathrm{C}$ & & & \\
\hline $\mathrm{C}$ & .000 & -2.64 & 7000 \\
\hline $\mathrm{C}$ & & -1.70 & \\
\hline $\mathrm{C}$ & -0.823 & & -2.6 \\
\hline $\mathrm{C}$ & 406000 & 2.04 & 3000 \\
\hline $\mathrm{C}$ & -0.984525000 & 2.44 & 78000 \\
\hline $\mathrm{C}$ & 1.217670000 & 3.40 & 4000 \\
\hline $\mathrm{C}$ & -0.080888000 & $0.83 \varepsilon$ & 7000 \\
\hline $\mathrm{C}$ & 2.132 & 1.775 & 7000 \\
\hline $\mathrm{C}$ & & -2.874 & 5000 \\
\hline $\mathrm{C}$ & 77000 & -1.934 & 2.230549000 \\
\hline $\mathrm{C}$ & & -3.475947000 & -1.554265000 \\
\hline $\mathrm{C}$ & & & 03000 \\
\hline $\mathrm{C}$ & -0.360816000 & -0.130060000 & -3.216529000 \\
\hline $\mathrm{C}$ & & & -3.485124000 \\
\hline $\mathrm{C}$ & -1.164927000 & 2.508519000 & -0.281211000 \\
\hline $\mathrm{C}$ & & & -0.514985000 \\
\hline $\mathrm{C}$ & -4.285497000 & 1.575731000 & 0.828739000 \\
\hline
\end{tabular}




\begin{tabular}{|c|c|c|c|}
\hline C & -4.679923000 & -0.689606000 & \\
\hline $\mathrm{N}$ & -5.121937000 & & 0.209449000 \\
\hline & -6.115760000 & & 0.311134000 \\
\hline & -5.381339000 & -1.901027000 & 0.193288000 \\
\hline & -5.535621000 & -1.948720000 & -1.134760000 \\
\hline & -5.842383000 & 40000 & 4000 \\
\hline & -6.154772000 & -3.006886000 & 18000 \\
\hline & -6.481324000 & & 9000 \\
\hline C & -6.645304000 & -4.068275000 & 91000 \\
\hline & -5.702980000 & -2.8 & 3000 \\
\hline & -6.261506000 & -3.007802000 & -2.73 \\
\hline & -6.846221000 & -4.819822000 & \\
\hline F & -7.13 & -4.9 & 2000 \\
\hline & -4.52 & & \\
\hline C & -4.5690 & 4.0 & 1.2 \\
\hline & -4.70 & & \\
\hline & -4.78 & 5.3 & 0.7 \\
\hline C & -4.9 & 4.3 & \\
\hline C & $-4.96 \xi$ & 5.5 & -0.6 \\
\hline $\mathrm{H}$ & -4.4 & & \\
\hline $\mathrm{H}$ & -4.82 & & 6000 \\
\hline & -5.0 & & 000 \\
\hline $\mathrm{H}$ & -5.1 & & -1.0 \\
\hline & -4.4 & & \\
\hline $\mathrm{H}$ & -4.8 & & \\
\hline \multicolumn{4}{|c|}{$\mathrm{Py}_{2} \mathrm{H}^{+} \mathrm{C}_{70} / \mathrm{b} 31 y p / 6-311 \mathrm{G}(\mathrm{d}, \mathrm{p})$} \\
\hline $\mathrm{C}$ & -0.672987000 & -0.076177000 & -3.4 \\
\hline $\mathrm{C}$ & -2.6 & -3.1 & \\
\hline $\mathrm{C}$ & -2.8 & -2.0 & \\
\hline $\mathrm{C}$ & -1.04 & & 000 \\
\hline $\mathrm{C}$ & & 2.9 & \\
\hline C & & 2.29 & -2.0 \\
\hline $\mathrm{C}$ & & -1.3 & -3.3 \\
\hline $\mathrm{C}$ & -2.992 & -3.28 & \\
\hline $\mathrm{C}$ & -2.388 & -0.80 & 3.32 \\
\hline $\mathrm{C}$ & -0.411478000 & 2.627 & 2.04 \\
\hline $\mathrm{C}$ & 2.5553 & 6000 & -0.36 \\
\hline $\mathrm{C}$ & & 862000 & -1.7 \\
\hline $\mathrm{C}$ & 000 & -2.65 & 5000 \\
\hline $\mathrm{C}$ & -5.096 & 0.8 & -1.58 \\
\hline $\mathrm{C}$ & 1.505576000 & -2.809 & 388000 \\
\hline $\mathrm{C}$ & -5.448665000 & 0.689976000 & -0.192085000 \\
\hline $\mathrm{C}$ & & -1.65 & 28000 \\
\hline $\mathrm{C}$ & -4.990692000 & 1.868764000 & 0.516349000 \\
\hline $\mathrm{C}$ & & -0.836265000 & 1.007615000 \\
\hline $\mathrm{C}$ & -4.356092000 & 2.750611000 & -0.444455000 \\
\hline $\mathrm{C}$ & & -0.643062000 & -1.474867000 \\
\hline $\mathrm{C}$ & -3.344126000 & 2.222242000 & -2.624029000 \\
\hline
\end{tabular}




\begin{tabular}{|c|c|c|c|}
\hline & 0.986277000 & & \\
\hline $\mathrm{C}$ & -4.662018000 & -0.267675000 & \\
\hline & 296078000 & -3.419961000 & .534977000 \\
\hline & .350823000 & -0.561735000 & 64000 \\
\hline & 1.202668000 & -1.113851000 & \\
\hline & -4.461057000 & 1.745297000 & 3000 \\
\hline & & 0.754514000 & \\
\hline & -3.220992000 & & 6000 \\
\hline & 1.3860 & -1.0975390 & \\
\hline & -2.893 & & \\
\hline & -0.275 & -3.73 & 2000 \\
\hline & -4.5 & -1.5 & \\
\hline & -0.50 & -2.8 & \\
\hline & -4.7 & -0.6 & \\
\hline & 1.022 & 0.3 & 2.9 \\
\hline $\mathrm{C}$ & -3.27 & & \\
\hline & 2.1 & & \\
\hline & -2.1 & & \\
\hline & 2.12 & & \\
\hline C & -2.1 & & \\
\hline & & -2.3 & \\
\hline & -3.5 & -0.1 & \\
\hline C & -0.6 & & \\
\hline & -4.8 & -1.7 & 000 \\
\hline C & -0.0 & & \\
\hline $\mathrm{C}$ & -4.3 & & \\
\hline $\mathrm{C}$ & 1.6 & & \\
\hline C & -2.67 & & \\
\hline $\mathrm{C}$ & 0.66 & & \\
\hline & -1.4 & & \\
\hline C & -1.27 & -3.3 & -1. \\
\hline $\mathrm{C}$ & -3.3 & -2.2 & \\
\hline $\mathrm{C}$ & -1.8 & -2.9 & \\
\hline $\mathrm{C}$ & -4.01 & -1.9 & \\
\hline$r$ & -0.33 & & \\
\hline $\mathrm{C}$ & -2.46 & 5000 & 9000 \\
\hline $\mathrm{C}$ & & & \\
\hline $\mathrm{C}$ & -0.879 & 3.5 & 1000 \\
\hline $\mathrm{C}$ & & 1.20 & \\
\hline $\mathrm{C}$ & -1.001 & 2.27 & -2.7 \\
\hline $\mathrm{C}$ & -0.649 & -2.44 & \\
\hline $\mathrm{C}$ & -2.76 & -1.3 & 42000 \\
\hline $\mathrm{C}$ & -1.967166000 & $-3.61 ?$ & 0.937188000 \\
\hline $\mathrm{C}$ & -4.08 & -2.5 & 000 \\
\hline $\mathrm{C}$ & -0.998547000 & -0.670726000 & 3.575635000 \\
\hline $\mathrm{C}$ & -3.123737000 & 0.393941000 & 3.179183000 \\
\hline $\mathrm{C}$ & 0.883757000 & & 1.569275000 \\
\hline $\mathrm{C}$ & -1.229266000 & & 1.155425000 \\
\hline $\mathrm{C}$ & 4.131472000 & 1.323331000 & 1.631729000 \\
\hline
\end{tabular}




\begin{tabular}{|c|c|c|c|}
\hline $\mathrm{C}$ & 4.440984000 & -1.086415000 & 1.443919000 \\
\hline $\mathrm{N}$ & 4.985482000 & & \\
\hline & 5.316231000 & & 2.850801000 \\
\hline & 5.286797000 & -1.702923000 & .337467000 \\
\hline & .931125000 & -0.875316000 & -0.505521000 \\
\hline & .432479000 & -3.075898000 & 1635000 \\
\hline $\mathrm{C}$ & 693525000 & -1.319154000 & -1.521115000 \\
\hline & .219540000 & -3.563088000 & 6000 \\
\hline $\mathrm{C}$ & .860870000 & -2.672331000 & -1.734443000 \\
\hline & 4.927064000 & -3.751406000 & 0.842503000 \\
\hline $\mathrm{H}$ & 7.159216000 & -0.562057000 & -2.137618000 \\
\hline & 6.336967000 & -4.631533000 & 71000 \\
\hline $\mathrm{H}$ & 000 & 4000 & 7000 \\
\hline 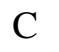 & 4.77 & & 7000 \\
\hline C & 9000 & 000 & 6000 \\
\hline $\mathrm{N}$ & 5.5 & & \\
\hline 0 & 8000 & 12000 & 2000 \\
\hline $\mathrm{C}$ & 5000 & & 8000 \\
\hline 0 & 564000 & 766000 & 0000 \\
\hline $\mathrm{H}$ & $3.9^{\prime}$ & 00 & \\
\hline $\mathrm{H}$ & 5.02 & & 3000 \\
\hline $\mathrm{H}$ & 00 & & 000 \\
\hline $\mathrm{H}$ & 00 & & 5000 \\
\hline $\mathrm{H}$ & 000 & & -0 . \\
\hline $\mathrm{H}$ & & & 000 \\
\hline $\mathrm{H}$ & 3.950947000 & 1.862265000 & 43000 \\
\hline \multicolumn{4}{|c|}{$\mathrm{Py}_{2} \mathrm{C}_{70} \mathrm{H}^{+} / \mathrm{b} 31 y p / 6-311 \mathrm{G}(\mathrm{d}, \mathrm{p})$} \\
\hline $\mathrm{C}$ & 2.099442000 & -0.705642000 & 3.416286000 \\
\hline $\mathrm{C}$ & & -3.3 & 1000 \\
\hline C & 000 & -1.5 & 5000 \\
\hline $\mathrm{C}$ & 00 & & \\
\hline $\mathrm{C}$ & 000 & 000 & 1000 \\
\hline $\mathrm{C}$ & & & 000 \\
\hline $\mathrm{C}$ & & & 22000 \\
\hline $\mathrm{C}$ & 25000 & -3.27116 & 45000 \\
\hline $\mathrm{C}$ & 858489000 & -0.255050000 & 580000 \\
\hline $\mathrm{C}$ & -0.174107000 & 2.914267000 & 48000 \\
\hline $\mathrm{C}$ & -2.197525000 & -1.486275000 & 1.743822000 \\
\hline $\mathrm{C}$ & 252000 & 1764000 & 9000 \\
\hline $\mathrm{C}$ & -1.716185000 & -2.682818000 & 876000 \\
\hline $\mathrm{C}$ & 5.434935000 & 0.514592000 & 0.047811000 \\
\hline $\mathrm{C}$ & -1.972085000 & -2.587481000 & -0.299940000 \\
\hline $\mathrm{C}$ & & & -1.374822000 \\
\hline $\mathrm{C}$ & -2.614145000 & -1.331447000 & -0.562472000 \\
\hline $\mathrm{C}$ & & 1.892114000 & -1.637583000 \\
\hline $\mathrm{C}$ & -3.049462000 & -0.680378000 & 0.758200000 \\
\hline $\mathrm{C}$ & & 2.595862000 & -0.375422000 \\
\hline $\mathrm{C}$ & -1.465993000 & -0.913279000 & 2.759419000 \\
\hline
\end{tabular}




\begin{tabular}{|c|c|c|c|}
\hline & 4.358928000 & 700498000 & \\
\hline & -0.554845000 & -3.312507000 & \\
\hline & 5.252127000 & -0.701383000 & \\
\hline & -1.054999000 & -3.129589000 & -1.205452000 \\
\hline & 4.753991000 & -0.520560000 & 570000 \\
\hline & -2.262171000 & -0.623282000 & -1.6 \\
\hline & 3.551216000 & 1.993391000 & \\
\hline & -2.791298000 & 0.918051000 & 9000 \\
\hline & 3.309116000 & 3.370227000 & \\
\hline & -0.249901000 & -1.53 & 3000 \\
\hline & 4.164501000 & 0.434736000 & 1000 \\
\hline & 0.401600000 & $-3.84 \mathrm{C}$ & \\
\hline$C$ & 7000 & -1.86 & \\
\hline 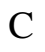 & -0.709291000 & -2.3937 & \\
\hline 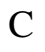 & 1000 & -0.41 & -3 . \\
\hline$C$ & -2.0 & & \\
\hline 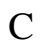 & 2.375586000 & 2.78 & -2.3 \\
\hline & -1.7 & & \\
\hline & 2.6 & & \\
\hline & -1.2 & & \\
\hline$C$ & 3.17 & 2.4 & \\
\hline & & -2.72 & \\
\hline 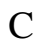 & 00 & -0.7 & \\
\hline & 00 & -3.7 & \\
\hline C & 4.5 & -1.7 & \\
\hline & -1.295 & -1.15 & \\
\hline C & & & \\
\hline & -2.16 & & \\
\hline$C$ & 2.25 & 3.4 & 000 \\
\hline & 0.701 & -0.50 & \\
\hline $\mathrm{C}$ & 2.88 & 0.4 & \\
\hline & & -3.6 & \\
\hline $\mathrm{C}$ & 00 & -2.6 & \\
\hline$r$ & & -2.5 & \\
\hline$C$ & 000 & -1.60 & 1000 \\
\hline $\mathrm{C}$ & -0.950 & 1.14 & \\
\hline$c$ & & & \\
\hline $\mathrm{C}$ & -0.935426000 & 2.406 & 5000 \\
\hline & & & \\
\hline $\mathrm{C}$ & 0.097574000 & 0.7623 & 50000 \\
\hline $\mathrm{C}$ & & & \\
\hline $\mathrm{C}$ & 1.605148000 & -2.911430000 & 99000 \\
\hline $\mathrm{C}$ & & -1.930 & 01000 \\
\hline $\mathrm{C}$ & 1.235008000 & -3.427588000 & 719000 \\
\hline $\mathrm{C}$ & 3.409696000 & -2.445498000 & 129000 \\
\hline $\mathrm{C}$ & -0.499926000 & -0.071628000 & \\
\hline $\mathrm{C}$ & 1.674489000 & 0.904040000 & -3.490052000 \\
\hline $\mathrm{C}$ & & & -0.102754000 \\
\hline $\mathrm{C}$ & 0.985607000 & 3.489525000 & -0.427663000 \\
\hline
\end{tabular}




$\begin{array}{lrrr}\mathrm{C} & -4.199461000 & 1.537451000 & 1.171042000 \\ \mathrm{C} & -4.585506000 & -0.849661000 & 1.075560000 \\ \mathrm{~N} & -5.174264000 & 0.485542000 & 0.747612000 \\ \mathrm{H} & -6.089211000 & 0.616741000 & 1.183338000 \\ \mathrm{C} & -5.336942000 & -1.917375000 & 0.304793000 \\ \mathrm{~N} & -5.905727000 & -1.490960000 & -0.827006000 \\ \mathrm{C} & -5.408232000 & -3.234128000 & 0.749337000 \\ \mathrm{C} & -6.561809000 & -2.379741000 & -1.578670000 \\ \mathrm{C} & -6.089091000 & -4.154941000 & -0.043498000 \\ \mathrm{C} & -6.673759000 & -3.723297000 & -1.229859000 \\ \mathrm{H} & -4.944322000 & -3.533907000 & 1.681258000 \\ \mathrm{H} & -7.013559000 & -1.998765000 & -2.488233000 \\ \mathrm{H} & -6.164799000 & -5.190789000 & 0.264631000 \\ \mathrm{H} & -7.213349000 & -4.408656000 & -1.871055000 \\ \mathrm{C} & -4.571931000 & 2.846695000 & 0.503449000 \\ \mathrm{C} & -4.251033000 & 4.078048000 & 1.066732000 \\ \mathrm{~N} & -5.217168000 & 2.715240000 & -0.659764000 \\ \mathrm{C} & -4.600184000 & 5.228604000 & 0.363534000 \\ \mathrm{C} & -5.553408000 & 3.824115000 & -1.324810000 \\ \mathrm{C} & -5.258857000 & 5.101856000 & -0.855257000 \\ \mathrm{H} & -3.741253000 & 4.139142000 & 2.020649000 \\ \mathrm{H} & -4.365542000 & 6.206931000 & 0.765419000 \\ \mathrm{H} & -6.078857000 & 3.680586000 & -2.262822000 \\ \mathrm{H} & -5.548952000 & 5.972729000 & -1.429248000 \\ \mathrm{H} & -5.350221000 & 0.553116000 & -0.274815000 \\ \mathrm{H} & -4.274409000 & 1.621798000 & 2.256419000 \\ \mathrm{H} & -4.701270000 & -0.993953000 & 2.151055000\end{array}$

